SERVIÇO DE PÓS-GRADUAÇÃO DO ICMC-USP

Data de Depósito:

Assinatura:

\title{
Equações polinomiais
}

\author{
Jonas Eduardo Carraschi
}

Orientador: Prof. Dr. Hermano de Souza Ribeiro

\begin{abstract}
Dissertação apresentada ao Instituto de Ciências Matemáticas e de Computação - ICMC-USP, como parte dos requisitos para obtenção do título de Mestre Programa de Mestrado Profissional em Matemática. VERSÃO REVISADA
\end{abstract}

USP - São Carlos

Maio de 2014 
Ficha catalográfica elaborada pela Biblioteca Prof. Achille Bassi e Seção Técnica de Informática, ICMC/USP, com os dados fornecidos pelo(a) autor(a)

\begin{tabular}{|c|c|}
\hline \multirow[t]{3}{*}{ c311e } & $\begin{array}{l}\text { Carraschi, Jonas Eduardo } \\
\quad \text { Equações polinomiais / Jonas Eduardo Carraschi; } \\
\text { orientador Hermano de Souza Ribeiro. -- São Carlos, } \\
2014 . \\
97 \mathrm{p} \text {. }\end{array}$ \\
\hline & $\begin{array}{l}\text { Dissertação (Mestrado - Programa de Pós-Graduação } \\
\text { em Mestrado Profissional em Matemática em Rede } \\
\text { Nacional) -- Instituto de Ciências Matemáticas e de } \\
\text { Computação, Universidade de São Paulo, } 2014 \text {. }\end{array}$ \\
\hline & $\begin{array}{l}\text { 1. Equações polinomiais. 2. Raízes de polinômios. } \\
\text { 3. Álgebra. 4. Ensino de Matemática. I. Ribeiro, } \\
\text { Hermano de Souza, orient. II. Título. }\end{array}$ \\
\hline
\end{tabular}


Dedico esse trabalho a todos os colegas e familiares, mas em especial aos meus avós que acabaram falecendo durante esse período, pois sempre me incentivaram e me aconselharam em relação aos estudos. 


\section{Agradecimentos}

Primeiramente a Deus, por propiciar-me a oportunidade de concluir o mestrado.

Ao orientador, Professor Dr. Hermano de Souza Ribeiro, pela paciência, pelo incentivo e pela dedicação junto à elaboração da dissertação.

Ao Professor Dr. Miguel, que ajudou muito na formatação do trabalho e na elaboração das figuras.

À Professora Coordenadora do Profmat, Ires Dias, pelos incentivos e conselhos.

Aos colegas de mestrado, pela amizade e companheirismo.

À minha família, pelo carinho, compreensão e incentivo durante os trabalhos. 


\section{Resumo}

Estudamos neste trabalho as equações polinomiais em sua abrangência: quadráticas, cúbicas e quárticas por diversos métodos clássicos, a limitação das raízes, resultados sobre equações polinomiais com coeficientes reais e inteiros, entre outros. 


\section{Abstract}

We studied in this work polynomial equations in a wide reach: quadratic, cubic and quartic polynomials by several classical methods, the boundness of roots, results about polynomial equations with real and integer coefficients, among other results. 


\section{Sumário}

\begin{tabular}{ll}
\hline Apresentação & 13
\end{tabular}

0 Métodos elementares de fatoração com completamento de quadrados e agrupamentos das potências pares e impares $\quad 15$

0.1 Equações Polinomiais com coeficientes reais . . . . . . . . . 15

$0.1 .1 \quad$ Sistema de numeração . . . . . . . . . . . . . . . . 15

0.1 .2 Determinação de taxas de juros . . . . . . . . . . . . . 16

$0.1 .3 \quad$ Problemas geométricos . . . . . . . . . . . . . . . . 17

0.1 .4 Equações polinomiais com coeficientes inteiros . . . . . 20

0.2 Agrupamento dos termos com potências pares e dos termos com potências ímpares . . . . . . . . . . . . . . . . . . 21

$0.2 .1 \quad$ Equações cúbicas . . . . . . . . . . . . . . . . . . . . . . 22

$0.2 .2 \quad$ Equações quárticas . . . . . . . . . . . . . . . . . . . . . . 23

$0.2 .3 \quad$ Completamento de quadrados . . . . . . . . . . . . . 24

0.2 .4 Fatoração do tipo $\left(A Z^{2}+B Z\right)^{2}+C\left(A Z^{2}+B Z\right)+D .25$

1 Resolução das equações polinomiais quadráticas, cúbicas e quárticas 27

1.1 Raízes das equações lineares afins . . . . . . . . . . . . . . . . 27

1.2 Raízes das equações quadráticas incompletas . . . . . . . . . . 27

1.3 Raízes das equações quadráticas completas: a fórmula de

Báskara ....................... 28

1.4 Raízes das equações polinomiais cúbicas: o método de Cardano 30

1.5 Raízes das equações cúbicas reduzidas com coeficientes

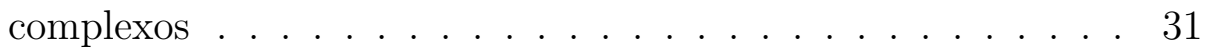

1.5.1 O método de Cardano . . . . . . . . . . . . . . . 31

1.5 .2 O método de Viète . . . . . . . . . . . . . . . . . . 33

1.5.3 O método de Gregory . . . . . . . . . . . . . . . . . 34

1.6 Raízes das equações polinomiais quárticas: o método de Ferrari 35 
1.7 Raízes das equações quárticas reduzidas com coeficientes

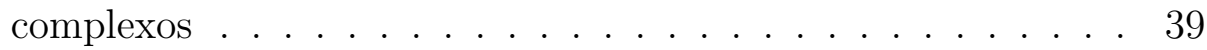

1.7.1 O método de Cartan-Ferrari . . . . . . . . . . . . . . . . 39

1.7 .2 O método de Gregory . . . . . . . . . . . . . . . . . . . . . . . . . . . . 40

1.7 .3 O método de Tschinhaus . . . . . . . . . . . . . . . . 41

\begin{tabular}{|ll|}
2 & Equações Polinomiais com coeficientes complexos \\
\hline
\end{tabular}

2.1 Teorema da Limitação das Raízes complexas . . . . . . . . . . 45

2.2 Teorema Fundamental da Álgebra . . . . . . . . . . . . . . . . 48

2.3 Relações de Girard . . . . . . . . . . . . . . . . . . . . . . . . . 48

2.4 Regra de Briot-Ruffini . . . . . . . . . . . . . . . . . . . . 51

2.5 Algoritmo de Peletarius . . . . . . . . . . . . . . . . . . 55

2.6 Fórmulas de Newton . . . . . . . . . . . . . . . . . . . . . . . 58

$\begin{array}{lll}3 & \text { Equações polinomiais com coeficientes reais } & 63\end{array}$

3.1 Teorema da limitação das raízes reais . . . . . . . . . . . . . 63

3.2 Teorema de Bolzano-Weierstrass . . . . . . . . . . . . . . . 68

3.2.1 Corolário do Teorema de Bolzano-Weierstrass . . . . . 72

3.3 Regra de sinais de Descartes . . . . . . . . . . . . 73

3.4 Teorema de Gua . . . . . . . . . . . . . . . . . . . . 74

3.5 Teorema de Rolle . . . . . . . . . . . . . . . . . . . . . . . . 77

3.6 Corolários do Teorema de Rolle . . . . . . . . . . . . . . . . . 78

3.7 Teorema de Sturm . . . . . . . . . . . . . . . . . . . . 80

\begin{tabular}{|lll}
\hline 4 & Equações Polinomiais com coeficientes inteiros & 87
\end{tabular}

4.1 Critério de irredutibilidade de Eiseinstein . . . . . . . . . . . . 87

4.2 Regras de exclusão de Newton . . . . . . . . . . . . . . . . . . . 90

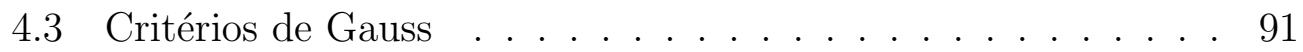




\section{Apresentação}

O tema Equações Polinomiais é frequente nos grandes concursos vestibulares do país em especial questões envolvendo as relações de Girard e a busca de soluções inteiras ou soluções racionais em equações polinomiais com coeficientes inteiros.

A dissertação aborda todos os tópicos sobre equações polinomiais contidas no Currículo Oficial da Secretaria de Educação do Estado de São Paulo para o Ensino Médio como Relações de Girard, Teorema de Raízes Racionais e o Algorítimo de Briot-Ruffini. A dissertação vai além, estudando temas avançados como o Teorema de Rolle para funções polinomiais, o Teorema e Bolzano Weierstrass, a Regra de Sinais de Descartes e o Teorema de Sturm, para funções polinomiais com raízes simples.

Um dos diferenciais da dissertação é que, na pesquisa de soluções inteiras em equações polinomiais com coeficientes inteiros recorda-se as regras de Gauss, as Regras de exclusão de Newton e o algorítimo de Peletarius derivado e Briot-Ruffini.

Outro diferencial é que na abordagem do algoritmo de Briot-Ruffini enfatizado o fato que mudanças lineares da variável transformam a equação polinomial dada em equações incompletas, ora desprovidas do termo de primeiro grau, ora desprovida do termo de segundo grau, ora do termo de terceiro grau e assim por diante e um dos pontos em destaque é a descrição do método Tschinhaus aplicado a equações cúbicas em que uma mudança de variável adequada anula simultaneamente todos os coeficientes intermediários de graus um e dois da equação cúbica dada.

Uma aula introdutória sobre equações polinomiais podem utilizar a parte envolvendo fatoração contida no capítulo zero e todo o último capítulo de equações polinomiais com coeficientes inteiros. Aplicações geométricas e sobre taxa de juros, que motivam o estudo das equações polinomiais, são também encontradas no capítulo zero. 
Uma aula interessante seria, além da resoluções de equações do primeiro e segundo grau as resoluções de equações cúbicas pelo Método de Cardano e da equações quárticas pelo Método de Cartan-Ferrari. Não existe um método geral para resolução das equações polinomiais com grau maior ou igual a 5 de acordo com a Teoria de Galois.

O Capítulo 1 contém os métodos de resoluções das raízes para equações quadráticas (Fórmula de Báskara), equações Cúbicas(Métodos de Cardano, de Viète, de Gregory e o método de Tschinhaus e equações quárticas (Método de Cartan-Ferrari e Gregory).

O Capítulo 2 trata das equações polinomiais com coeficientes complexos com destaque para o Teorema das Limitações das Raízes Complexas, do Teorema Fundamental da Álgebra, Relações de Girard, a Regra de BriotRuffini, o Algoritmo de Peletarius e as Fórmulas de Newton.

O Capítulo 3, trata das equações polinomiais com coeficientes reais, com destaque para o Teorema da limitação das Raízes Reais, o Teorema de Bolzano, a Regra de Sinais de Descartes, o Teorema de Gua, o Teorema de Rolle e o Teorema da Separação das Raízes de Sturm.

O Capítulo 4 estuda as equações polinomiais com coeficientes inteiros, com destaque para o Critério de Irredutibilidade de Eiseinstein além das Regras de Gauss, das Regras de Exclusão de Newton e do Teorema das Raízes Fracionárias. 


\section{Capítulo 0}

\section{Métodos elementares de fatoração com completamento de quadrados e agrupamentos das potências pares e impares}

\subsection{Equações Polinomiais com coeficientes reais}

A resolução de equações polinomiais com coeficientes reais e grau superior ou igual a um ocorre em várias situações:

\subsubsection{Sistema de numeração}

Exemplo 0.1. Qual é a base $b$ do sistema de numeração no qual o número cuja representação no sistema de numeração decimal é 538, é representado por 4123 no sistema de numeração com base $b$ ?

Resolução. A equação polinomial cuja raiz inteira positiva é $b$ é dada por

$$
4 x^{3}+x^{2}+2 x+3=538
$$

isto é,

$$
4 b^{3}+b^{2}+2 b=535
$$

$\mathrm{Ou}$

$$
b\left(4 b^{2}+b+2\right)=535
$$


o que mostra que $535=5 \cdot 107$ é um múltiplo inteiro do número natural $b$.

Por inspeção, a única resposta do problema é 5, visto que 107 obviamente não é raiz da equação cúbica

$$
4 x^{3}+x^{2}+2 x=535 .
$$

\subsubsection{Determinação de taxas de juros}

Exemplo 0.2. Uma dívida de 1000 reais deve ser paga em duas vezes: a primeira parcela deve ser paga 90 dias após o empréstimo no valor de 600 reais e a segunda parcela no valor de 700 reais a ser paga doze meses após a data da dívida. Qual é a taxa mensal de juros $I$ associada a dívida?

Resolução. Temos que

$$
1000=\frac{600}{(1+I)^{3}}+\frac{700}{(1+I)^{12}}
$$

$\mathrm{Ou}$

$$
10(1+I)^{12}=6(1+I)^{9}+7,
$$

isto é, $1+I$ é a raiz da equação polinomial com coeficientes inteiros

$$
P(x)=10 x^{12}-6 x^{9}-7=0,
$$

a qual tem que admitir uma única raiz real maior do que um.

A regra de sinal de Descartes a ser estudada na Seção 3.3 garante a existência de uma única raiz real positiva devido ao número de variações de sinais dos coeficientes da equação

$$
10 ;-6 ;-7
$$

ser igual a um.

O teorema de Bolzano-Weierstrass do mesmo Capítulo 3 garante a existência de uma raiz real compreendida entre 1 e 2 , pois $P(1) \cdot P(2)<0$

A resposta exata para a questão da determinação de taxa mensal de juros I não é obtida apesar de se tratar de uma equação polinomial com coeficientes inteiros. 
Adiantando que raízes fracionárias da equação $10 x^{12}-6 x^{9}-7=0$ de forma $\frac{p}{q} \operatorname{com} p, q \in \mathbb{Z}, q \neq 0$, sendo $p$ e $q$ primos entre si, temos:

$$
10\left(\frac{p}{q}\right)^{12}-6\left(\frac{p}{q}\right)^{9}=7
$$

$\mathrm{Ou}$

$$
10 p^{12}-6 p^{9} q^{3}=7 q^{12}
$$

isto é, $p$ é um divisor inteiro de 7 e analogamente, $q$ é um divisor de 10.

Portanto, $\frac{p}{q}$ só pode ser $1,7, \frac{1}{2}, \frac{7}{2}, \frac{1}{5}, \frac{7}{5}, \frac{1}{10}, \frac{7}{10}$.

Analisando os valores, o único valor de $\frac{p}{q}$ compreendido entre 1 e 2 é $\frac{7}{5}$, o qual não é raiz da equação.

Refinando o resultado, temos que $P(1) \cdot P\left(\frac{11}{10}\right)<0$, portanto a raiz está entre 1 e $\frac{11}{10}$ e com isso a taxa de juros é menor que $10 \%$.

\subsubsection{Problemas geométricos}

Exemplo 0.3. Dadas três retas paralelas no plano, em que a distância entre as duas primeiras é $a$, sendo $a>0$, e a distância entre as duas últimas é $b$, sendo $b>0$. Qual é o valor do lado do triângulo equilátero, cujos vértices $A, B$ e $C$ pertencem a uma e apenas a uma das retas paralelas?

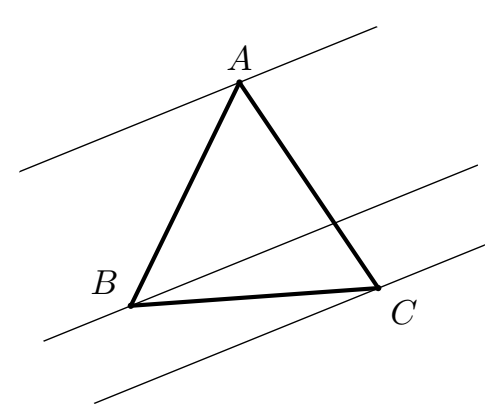

Resolução. Construindo um sistema ortogonal de coordenadas cartesianas, cuja origem é o vértice $B$ pertencente a segunda reta paralela e cuja a reta das abscissas é a reta paralela por $B$, os demais vértices $A$ e $C$ do triângulo equilátero a serem construídos tem coordenadas $(x, a)$ e $(y,-b)$ respectivamente.

Então a medida ao quadrado do segmento $A B$ é $x^{2}+a^{2}$, a medida ao quadrado do segmento $B C$ é $y^{2}+b^{2}$ e a medida ao quadrado do segmento $A C$ é $(x-y)^{2}+(a+b)^{2}$. 
Impondo que

$$
x^{2}+a^{2}=y^{2}+b^{2}=(x-y)^{2}+(a+b)^{2}
$$

vem

$$
x^{2}-2 x y+y^{2}+a^{2}+2 a b+b^{2}=y^{2}+b^{2}
$$

$\mathrm{Ou}$

$$
x^{2}-2 x y+a^{2}+2 a b=0
$$

ou

$$
\begin{gathered}
4 x^{2} y^{2}=\left(x^{2}+a^{2}+2 a b\right)^{2} \\
4 x^{2}\left(x^{2}+a^{2}-b^{2}\right)=\left(x^{2}+a^{2}+2 a b\right)^{2}
\end{gathered}
$$

até a obtenção da equação biquadrada

$$
3 x^{4}+\left(2 a^{2}-4 b^{2}-4 a b\right) x^{2}-\left(a^{4}+4 a^{2} b^{2}+4 a^{3} b\right)=0
$$

cujo discriminante é

$$
16\left[\left(a^{2}+b^{2}\right)^{2}+a b\left(a b+2 a^{2}+2 b^{2}\right)\right]
$$

o qual é positivo o que mostra que a equação biquadrada tem uma raiz positiva, e uma raiz negativa (o produto das raízes é o termo independente da equação devido as relações de Girard a ser visto no capítulo dois) e um par de raízes complexas conjugadas.

A única raiz positiva $x$ da equação biquadrada origina a solução para o problema geométrico: $\sqrt{x^{2}+a^{2}}$ é a medida do lado do triângulo equilátero.

A única raiz negativa $x$ da equação biquadrada origina a outra solução do triângulo equilátero, cuja medida do lado é a mesma do caso da raiz positiva.

Caso $a=b$,

$$
x^{2}+a^{2}=y^{2}+b^{2}=(x-y)^{2}+(a+b)^{2}
$$

é fácil determinar a media do lado do triângulo equilátero a saber $\sqrt{3} a$. $\diamond$

Exemplo 0.4. Dado dois triângulos isósceles $\triangle A B C$ e $\triangle C D E$, cujas bases $A C$ e $C E$ estão sobre a mesma reta, cujas medidas dos lados iguais dos dois triângulos são iguais $A B=B C=C D=D E$ e de modo que o vértice $D$ do segundo triângulo pertence a reta suporte do lado $A B$ do primeiro triângulo, o objetivo é o cálculo da medida $x$ da base $A C$. 


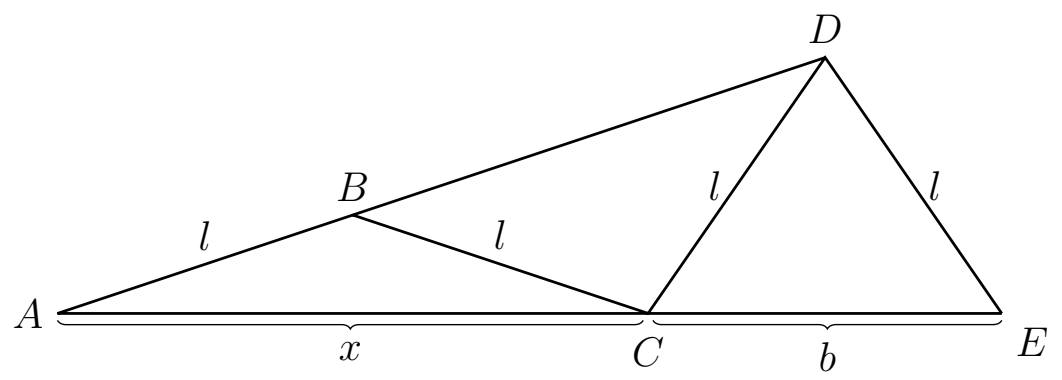

Resolução. O objetivo é a determinação da medida $x$ da base $A C$ em termos da medida $b$ da base $C E$ e em termos da medida $l$ dos lados congruentes $A B=B C=C D=D E$.

Para o cálculo de $x$, considere a circunferência com centro $C$ e raio $l$ e sejam $H$ e $K$ os pontos de intersecção da circunferência com a reta das bases. Seja $F$ e $G$ respectivamente os pontos de intersecção das retas perpendiculares a reta das bases traçadas por $\mathrm{B}$ e $\mathrm{D}$.

Portanto

$\mathrm{e}$

$$
A F=F C=\frac{x}{2}
$$

$$
F G=\frac{b+x}{2}
$$

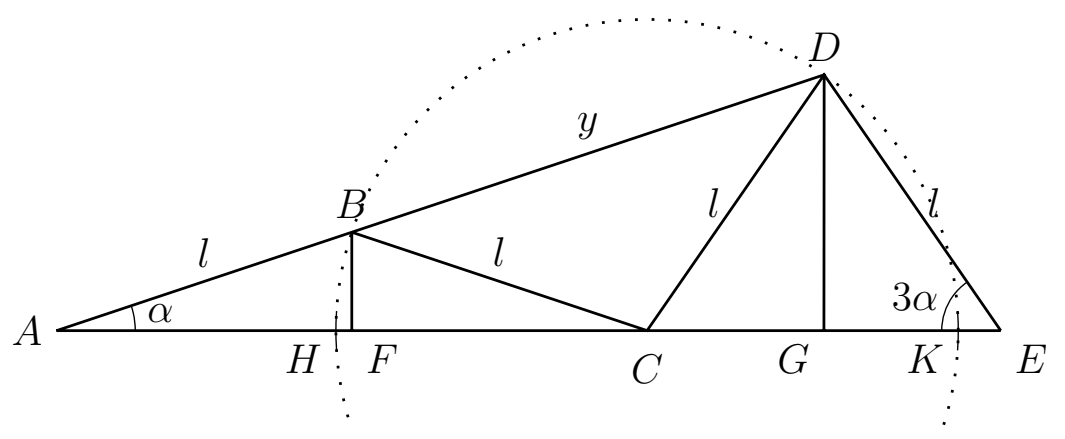

Se $y=B D$ pela semelhança de triângulos entre o $\triangle A B F$ e $\triangle A D G$, temos:

$$
\frac{y}{l}=\frac{\frac{b+x}{2}}{\frac{x}{2}}
$$

isto é,

$$
\frac{y}{l}=\frac{b+x}{x}
$$


que é equivalente a

$$
x y=l(x+b) .
$$

Por outro lado, considerando a potência do vértice $A$ em relação a circunferência traçada

$$
\begin{aligned}
A H \cdot A K & =l(y+l) \\
& =(x-l)(x+l)=x^{2}-l^{2}
\end{aligned}
$$

a eliminação de $y$ nas duas ultimas equações conduz a

$$
x^{3}-3 l^{2} x=b l^{2}
$$

Sendo o ângulo $F \hat{A} B=\alpha$, e calculando $\cos (a)=\frac{x}{2 l}$ e identificando que $\cos (3 \alpha)=\frac{b}{2 l}$, a equação polinomial em $x$

$$
x^{3}-3 l^{2} x=b l^{2}
$$

é equivalente a

$$
\cos (3 \alpha)=4 \cos ^{3}(\alpha)-3 \cos (\alpha)
$$

que é a formula do ângulo triplo da trigonometria.

Uma aplicação da fórmula do ângulo triplo é que equações cúbicas com coeficientes reais da forma

$$
x^{3}-3 x-a=0
$$

com $|a|<2$ admite como raiz $2 \cos \left(\frac{\theta}{3}\right)$ desde que $\cos (\theta)=\frac{a}{2}$.

$\diamond$

\subsubsection{Equações polinomiais com coeficientes inteiros}

Exemplo 0.5. Equações sexticas, como por exemplo, a equação

$$
x^{6}-2.6 x+91=0
$$

não admite raízes reais pois, para $x>0$, devido a desigualdade estrita entre as médias aritmética e geométrica

$$
\frac{x^{6}+2^{6}+1+1+1+1}{6}>\sqrt[6]{x^{6} \cdot 2^{6} \cdot 1 \cdot 1 \cdot 1 \cdot 1}=2 x
$$


pois

$$
\begin{gathered}
x^{6}+91=x^{6}+2^{6}+1+1+1+1+23 \\
>x^{6}+2^{6}+1+1+1+1 \\
>6.2 x
\end{gathered}
$$

o que mostra que para $x>0$

$$
x^{6}-2.6 x+91>0
$$

e ainda se $x<0$

$$
(-x)^{6}-2.6(-x)+91>91
$$

o que prova que a equação

$$
x^{6}-12 x+91=0
$$

admite apenas três pares de raízes complexas conjugadas duas a duas.

Com raciocínio análogo, é fácil construir outras equações polinomiais com coeficientes inteiros, que não admitem raízes reais, como por exemplo:
I) $x^{6}-3.6 x+751=0$
II) $x^{4}-2.4 x+43=0$
III) $x^{4}-3.4 x+119=0$

\subsection{Agrupamento dos termos com potências pares e dos termos com potências ímpares}

Dada uma equação polinomial de grau $N \geqslant 1$ com coeficientes inteiros

$$
P(Z)=Z^{N}+A_{N-1} Z^{N-1}+\ldots+A_{1} Z+A_{0}=0
$$

o objetivo é a determinação das $N$ raízes da equação contadas as suas multiplicidades.

A técnica da fatoração é a primeira tentativa para determinação das raízes da equação $P(Z)=0$ 
Exemplo 0.6.

$$
\begin{gathered}
Z^{6}+2 Z^{5}+10 Z^{4}-Z^{2}-2 Z-10=0 \\
\left(Z^{6}+10 Z^{4}-Z^{2}-10\right)+\left(2 Z^{5}-2 Z\right)=0 \\
Z^{4}\left(Z^{2}+10\right)-1\left(Z^{2}+10\right)+2 Z\left(Z^{4}-1\right)=0 \\
\left(Z^{2}+10\right)\left(Z^{4}-1\right)+2 Z\left(Z^{4}-1\right)=0 \\
\left(Z^{4}-1\right)\left(Z^{2}+10+2 Z\right)=0 \\
\left(Z^{2}+1\right)\left(Z^{2}-1\right)(Z+1+3 i)(Z+1-3 i)=0 \\
(Z+i)(Z-i)(Z-1)(Z+1)(Z+1+3 i)(Z+1-3 i)=0
\end{gathered}
$$

logo temos que $i,-i,-1-3 i,-1+3 i, 1, \mathrm{e}-1$ são as raízes da equação $Z^{6}+2 Z^{5}+10 Z^{4}-Z^{2}-2 Z-10=0$.

\subsubsection{Equações cúbicas}

Exemplo 0.7.

$$
\begin{gathered}
64 Z^{3}-16 Z^{2}+12 Z-3=0 \\
\left(64 Z^{3}+12 Z\right)-\left(16 Z^{2}+3\right)=0 \\
4 Z\left(16 Z^{2}+3\right)-1\left(16 Z^{2}+3\right)=0 \\
\left(16 Z^{2}+3\right)(4 Z-1)=0 \\
4\left(Z+\frac{\sqrt{3} i}{4}\right)\left(Z-\frac{\sqrt{3} i}{4}\right)\left(Z-\frac{1}{4}\right)=0
\end{gathered}
$$

portanto, $\frac{\sqrt{3} i}{4},-\frac{\sqrt{3} i}{4}$ e $\frac{1}{4}$ são raízes da equação $64 Z^{3}-16 Z^{2}+12 Z-3=0$.

Exemplo 0.8.

$$
\begin{gathered}
3 Z^{3}+2 Z^{2}-3 Z-2=0 \\
\left(3 Z^{3}-3 Z\right)+\left(2 Z^{2}-2\right)=0 \\
3 Z\left(Z^{2}-1\right)+2\left(Z^{2}-1\right)=0 \\
\left(Z^{2}-1\right)(3 Z+2)=0 \\
\left(Z^{2}-1\right)(3 Z+2)=0 \\
3(Z-1)(Z+1)\left(Z+\frac{2}{3}\right)=0
\end{gathered}
$$

$\operatorname{logo} 1,-1$ e $\frac{2}{3}$ são raízes da equação $3 Z^{3}+2 Z^{2}-3 Z-2=0$. 


\subsubsection{Equações quárticas}

\section{Fatoração de equações quárticas em equações quadráticas}

Exemplo 0.9.

$$
\begin{gathered}
Z^{4}-4 Z^{3}+6 Z^{2}-4 Z+5=0 \\
\left(Z^{4}+6 Z^{2}+5\right)-4 Z\left(Z^{2}+1\right)=0 \\
\left(Z^{4}+Z^{2}\right)+\left(5 Z^{2}+5\right)-4 Z\left(Z^{2}+1\right)=0 \\
\left(Z^{2}\right)\left(Z^{2}+1\right)+5\left(Z^{2}+1\right)-4 Z\left(Z^{2}+1\right)=0 \\
\left(Z^{2}+1\right)\left(Z^{2}-4 Z+5\right)=0 \\
(Z-i)(Z+i)(Z-2-i)(Z-2+i)=0
\end{gathered}
$$

O último fator em (1) é obtido pelo completamento de quadrados:

$$
\begin{gathered}
Z^{2}-4 Z+5=0 \\
Z^{2}-4 Z+4=-1 \\
(Z-2)^{2}=i^{2} \\
Z=2 \pm i
\end{gathered}
$$

portanto podemos afirmar que $2+i, 2-i,-i$ e $+i$ são as raízes da equação $Z^{4}-4 Z^{3}+6 Z^{2}-4 Z+5=0$

Exemplo 0.10 .

$$
\begin{gathered}
Z^{4}-6 Z^{3}+3 Z^{2}+24 Z-28=0 \\
\left(Z^{4}+3 Z^{2}-28\right)+\left(-6 Z^{3}+24 Z\right)=0 \\
\left(Z^{4}+3 Z^{2}-28\right)-6 Z\left(Z^{2}-4\right)=0 \\
\left(Z^{2}-4\right)\left(Z^{2}+7\right)-6 Z\left(Z^{2}-4\right)=0 \\
\left(Z^{2}-4\right)\left(Z^{2}-6 Z+7\right)=0 \\
(Z-2)(Z+2)(Z-3+\sqrt{2})(Z-3-\sqrt{2})=0
\end{gathered}
$$

Os últimos dois fatores de (2) são obtidos pelo completamento de quadrados:

$$
\begin{gathered}
Z^{2}-6 Z+9=2 \\
(Z-3)^{2}=2 \\
Z=3 \pm \sqrt{2}
\end{gathered}
$$


$\operatorname{logo}, 3+\sqrt{2}, 3-\sqrt{2}, 2$ e -2 são raízes da equação $Z^{4}-6 Z^{3}+3 Z^{2}+24 Z-28=$ 0 .

Exemplo 0.11. Resolução de uma equação de quinto grau.

$$
\begin{gathered}
Z^{5}+5 Z^{3}-7 Z^{2}-35=0 \\
\left(Z^{5}+5 Z^{3}\right)-\left(7 Z^{2}+35\right)=0 \\
Z^{3}\left(Z^{2}+5\right)-7\left(Z^{2}+5\right)=0 \\
\left(Z^{2}+5\right)\left(Z^{3}-7\right)=0 \\
\left(Z^{2}+5\right)(Z-\sqrt[3]{7})\left(Z^{2}+\sqrt[3]{7} Z+\sqrt[3]{49}\right)=0
\end{gathered}
$$

cujas raízes são

$$
5 i,-5 i, \sqrt[3]{7},-\frac{\sqrt[3]{7}}{2}-\frac{\sqrt{3}}{2} \sqrt[3]{7} i,-\frac{\sqrt[3]{7}}{2}+\frac{\sqrt{3}}{2} \sqrt[3]{7} i
$$

As duas últimas são obtidas com o completamento de quadrados:

$$
\begin{gathered}
Z^{2}+\sqrt[3]{7} Z+\sqrt[3]{49} \\
\left(Z^{2}+\frac{2 \sqrt[3]{7}}{2} Z+\frac{\sqrt[3]{49}}{4}\right)+\frac{3}{4} \sqrt[3]{49}=0
\end{gathered}
$$

que equivale a

$$
\left(Z+\frac{\sqrt[3]{7}}{2}\right)^{2}=-\frac{3}{4} \sqrt[3]{49} i^{2}
$$

cujas raízes são

$$
Z=-\frac{\sqrt{7}}{2} \pm \frac{\sqrt{3}}{2} \sqrt[3]{7} i
$$

\subsubsection{Completamento de quadrados}

No caso de equações quadráticas, o completamento de quadrados conduz a obtenção da fórmula de Báskara:

$$
\begin{gathered}
A Z^{2}+B Z+C=0, \quad A \neq 0 \\
A\left[Z^{2}+2 \frac{B}{2 A} Z+\frac{B^{2}}{4 A^{2}}\right]=-C+\frac{B^{2}}{4 A} .
\end{gathered}
$$

Quando os coeficientes são complexos, a propriedade da equação quadrática ter raízes complexas conjugadas não é válida: $i$ e $1+i$ são raízes de $Z^{2}-(1+2 i) Z+(-1+i)=0$ e os números complexos $i$ e $1+i$ não são complexos conjugados. 
Exemplo 0.12. O completamento de quadrados tem aplicações em equações de grau superior a dois como na equação quártica:

$$
\begin{gathered}
Z^{4}-6 Z^{3}+8 Z^{2}+2 Z-1=0 \\
Z^{4}-6 Z^{3}+9 Z^{2}=9 Z^{2}-8 Z^{2}-2 Z+1 \\
Z^{4}-6 Z^{3}+9 Z^{2}=Z^{2}-2 Z+1 \\
\left.Z^{2}-3 Z\right)^{2}=(Z-1)^{2} \\
\left(Z^{2}-3 Z\right)^{2}-(Z-1)^{2}=0 \\
{\left[\left(Z^{2}-3 Z\right)+(Z-1)\right] \cdot\left[\left(Z^{2}-3 Z\right)-(Z-1)\right]=0} \\
\left(Z^{2}-4 Z+1\right)\left(Z^{2}-2 Z-1\right)=0 .
\end{gathered}
$$

Temos

$$
\begin{gathered}
Z^{2}-4 Z+1=0 \\
Z^{2}-4 Z+4=3 \\
(Z-2)^{2}=3 \\
Z=2 \pm \sqrt{3}
\end{gathered}
$$

e

$$
\begin{gathered}
Z^{2}-2 Z-1=0 \\
Z^{2}-2 Z+1=2 \\
(Z-1)^{2}=2 \\
Z=1 \pm \sqrt{2} .
\end{gathered}
$$

Logo as raízes da equação (3) são $2+\sqrt{3}, 2-\sqrt{3}, 1-\sqrt{2}$ e $1+\sqrt{2}$.

\subsubsection{Fatoração do tipo $\left(A Z^{2}+B Z\right)^{2}+C\left(A Z^{2}+B Z\right)+D$}

Exemplo 0.13 .

$$
\begin{gathered}
Z^{6}-8 Z^{5}+25 Z^{4}-36 Z^{3}+20 Z^{2}=0 \\
Z^{2}\left[Z^{4}-8 Z^{3}+25 Z^{2}-36 Z+20\right]=0 \\
Z^{2}\left[Z^{4}-8 Z^{3}+16 Z^{2}+9 Z^{2}-36 Z+20\right]=0 \\
Z^{2}\left[\left(Z^{2}-4 Z\right)^{2}+9\left(Z^{2}-4 Z\right)+20\right]=0
\end{gathered}
$$


nesse caso temos que $\left(Z^{2}-4 Z\right)$ é raiz da equação

$$
\left(Z^{2}-4 Z\right)^{2}+9\left(Z^{2}-4 Z\right)+20=0
$$

portanto,

$$
\begin{gathered}
Z^{2}\left(Z^{2}-4 Z+5\right)\left(Z^{2}-4 Z+4\right)=0 \\
Z^{2}(Z-2-i)(Z-2+i)(Z-2)^{2}=0
\end{gathered}
$$

logo $0,2+i, 2-i$ e 2 são as raízes da equação $Z^{6}-8 Z^{5}+25 Z^{4}-36 Z^{3}+20 Z^{2}=$ 0 .

Exemplo 0.14 .

$$
\begin{gathered}
Z^{3}+4 Z^{2}+8 Z+8=0 \\
\left(Z^{3}+8\right)+\left(4 Z^{2}+8 Z\right)=0 \\
\left(Z^{3}+2^{3}\right)+4 Z(Z+2)=0 \\
(Z+2)\left(Z^{2}-2 Z+4\right)+4 Z(Z+2)=0 \\
(Z+2)\left(Z^{2}-2 Z+4+4 Z\right)=0 \\
(Z+2)\left(Z^{2}+2 Z+4\right)=0 \\
(Z+2)(Z+1-\sqrt{3} i)(Z+1+\sqrt{3} i)=0 .
\end{gathered}
$$

Nesse caso, temos que $-2,-1-\sqrt{3} i$ e $-1+\sqrt{3} i$ são as raízes da equação $Z^{3}+4 Z^{2}+8 Z+8=0$.

Exemplo 0.15.

$$
\begin{gathered}
Z^{3}-2 Z+4=0 \\
\left(Z^{3}+8\right)-2 Z-4=0 \\
\left(Z^{3}+2^{3}\right)-2(Z+2)=0 \\
(Z+2)\left(Z^{2}-2 Z+4\right)-2(Z+2)=0 \\
(Z+2)\left(Z^{2}-2 Z+4-2\right)=0 \\
(Z+2)\left(Z^{2}-2 Z+2\right)=0 \\
(Z+2)(Z-1-i)(Z-1+i)=0 .
\end{gathered}
$$

Portanto temos que $-2,1+i$ e $1-i$ são as raízes da equação $Z^{3}-2 Z+4=$ 0 . 


\section{Capítulo 1}

\section{Resolução das equações polinomiais quadráticas, cúbicas e quárticas}

\subsection{Raízes das equações polinomiais lineares afins}

A equação polinomial com coeficientes complexos e com grau um representada por

$$
\begin{gathered}
P(Z)=A Z+B=0 \\
A, B \in \mathbb{C}, \quad A \neq 0
\end{gathered}
$$

admite uma única raiz complexa a saber $\frac{-B}{A}$, isto é, $\frac{-B}{A}$ é o único número complexo com a propriedade $P\left(\frac{-B}{A}\right)=0$.

\subsection{Raízes das equações polinomiais quadráticas incompletas}

A equação polinomial quadrática incompleta com coeficientes complexos

$$
\begin{gathered}
Z^{2}=a+i b \\
a, b \in \mathbb{R}, \quad a^{2}+b^{2} \neq 0
\end{gathered}
$$

admite duas raízes complexas obtidas da seguinte maneira: se $x$ e $y$ indicarem as partes real e imaginária do número complexo $Z$ então

$$
Z=x+i y
$$




$$
Z^{2}=\left(x^{2}-y^{2}\right)+i(2 x y)=a+i b
$$

e, pela igualdade entre as partes real e imaginária dos dois membros da última equação,

$$
\begin{gathered}
x^{2}-y^{2}=a, \\
2 x y=b .
\end{gathered}
$$

De $\left(x^{2}+y^{2}\right)^{2}=\left(x^{2}-y^{2}\right)^{2}+4 x^{2} y^{2}=a^{2}+b^{2}$ vem

$$
x^{2}+y^{2}=\sqrt{a^{2}+b^{2}}
$$

portanto,

$$
\begin{aligned}
& x^{2}=\frac{a+\sqrt{a^{2}+b^{2}}}{2}, \\
& y^{2}=\frac{-a+\sqrt{a^{2}+b^{2}}}{2} .
\end{aligned}
$$

e os sinais de $x$ e de $y$ devem respeitar a condição $2 x y=b$.

Temos assim que as raízes quadradas do número complexo $a+b i$ são definidos por

$$
\pm \sqrt{\frac{a+\sqrt{a^{2}+b^{2}}}{2}} \pm i \sqrt{\frac{-a+\sqrt{a^{2}+b^{2}}}{2}}
$$

em que os sinais das partes real e imaginária devem ser compatíveis com o sinal de $b$.

\subsection{Raízes das equações polinomiais quadráticas completas: a fórmula de Báskara}

A fórmula de Báskara, obtida pela técnica do completamento de quadrados, exibe as raízes complexas da equação polinomial quadrática com coeficientes complexos

$$
\begin{gathered}
P(Z)=A Z^{2}+B Z+C=0 \\
\text { em que } A, B, C \in \mathbb{C}, A \neq 0
\end{gathered}
$$


De fato,

$$
\begin{gathered}
A\left[Z^{2}+2 \frac{B}{2 A} Z+\frac{B^{2}}{4 A^{2}}\right]=\frac{B^{2}}{4 A}-C \\
\left(Z+\frac{B}{2 A}\right)^{2}=\frac{B^{2}-4 A C}{4 A^{2}}
\end{gathered}
$$

A última equação é uma equação quadrática incompleta

$$
z^{2}=\frac{B^{2}-4 A C}{4 A^{2}}
$$

obtida pela mudança de variável

$$
z=Z+\frac{B}{2 A}
$$

Então,

$$
\begin{aligned}
& Z_{1}=\frac{-B+\sqrt{B^{2}-4 A C}}{2 A} \\
& Z_{2}=\frac{-B-\sqrt{B^{2}-4 A C}}{2 A}
\end{aligned}
$$

são os dois números complexos não necessariamente distintos com a propriedade

$$
P\left(Z_{1}\right)=0 \quad \text { e } P\left(Z_{2}\right)=0 .
$$

As relações entre as raízes $Z_{1}$ e $Z_{2}$ da equação quadrática e os coeficientes $A, B$ e $C$ da equação quadrática são conhecidas como relações de Girard

$$
\begin{aligned}
Z_{1}+Z_{2} & =-\frac{B}{A} \\
Z_{1} Z_{2} & =\frac{C}{A}
\end{aligned}
$$

Além disso, o discriminante da equação quadrática definido por

$$
\left(Z_{1}-Z_{2}\right)^{2}=\left(Z_{1}+Z_{2}\right)^{2}-4 Z_{1} Z_{2}=\frac{B^{2}-4 A C}{A^{2}}
$$

mostra que a equação quadrática admite uma única raiz complexa, a saber $-B / 2 A$, no caso em que $B^{2}-4 A C=0$. 
Em consequência, vem a fatoração da equação polinomial quadrática em fatores lineares

$$
\begin{aligned}
P(Z)=A Z^{2}+ & B Z+C \\
& =A\left[Z-\frac{-B+\sqrt{B^{2}-4 A C}}{2 A}\right]\left[Z-\frac{-B-\sqrt{B^{2}-4 A C}}{2 A}\right]
\end{aligned}
$$

\subsection{Raízes das equações polinomiais cúbicas: o método de Cardano}

A equação polinomial cúbica completa com coeficientes complexos

$$
\begin{gathered}
P(Z)=A Z^{3}+B Z^{2}+C Z+D=0 \\
\quad \text { em que } A, B, C, D \in \mathbb{C}, A \neq 0
\end{gathered}
$$

admite as mesmas raízes da equação cúbica mônica, isto é, com coeficiente de $Z^{3}$ igual a um, ou seja

$$
Z^{3}+\frac{B}{A} Z^{2}+\frac{C}{A} Z+\frac{D}{A}=0 .
$$

A equação cúbica incompleta reduzida da equação dada é obtida pela mudança canônica de variável

$$
Z=z-\frac{B}{3 A}
$$

e é dada por

$$
z^{3}+a z+b=0
$$

com

$$
\begin{aligned}
a & =\frac{C}{A}-\frac{1}{3}\left(\frac{B}{A}\right)^{2} \\
b & =\frac{2}{27}\left(\frac{B}{A}\right)^{3}-\frac{1}{3}\left(\frac{B}{A}\right)\left(\frac{C}{A}\right)+\frac{D}{A}
\end{aligned}
$$

Os discriminantes da equação cúbica completa e da equação cúbica reduzida associada são iguais a

$$
\left(z_{1}-z_{2}\right)^{2}\left(z_{1}-z_{3}\right)^{2}\left(z_{2}-z_{3}\right)^{2}=-4 a^{3}-27 b^{2}
$$


em que $z_{1}, z_{2}$ e $z_{3}$ são as raízes da equação reduzida

$$
z^{3}+a z+b=0
$$

Tendo em vista as relações de Girard

$$
\begin{gathered}
z_{1}+z_{2}+z_{3}=0 \\
z_{1} z_{2}+z_{1} z_{3}+z_{2} z_{3}=a \\
z_{1} z_{2} z_{3}=-b
\end{gathered}
$$

O discriminante de uma equação cúbica é um polinômio simétrico nas raízes da equação e como tal é expresso em termos dos coeficientes da equação: o discriminante de

$$
Z^{3}-s_{1} Z^{2}+s_{2} Z-s_{3}=0
$$

é igual a

$$
-4 s_{1}^{3}+s_{1}^{2}+18 s_{1} s_{2} s_{3}-4 s_{2}^{3}-27 s_{3}^{2}
$$

que no caso da equação reduzida $z^{3}+a z+b=0$, é igual a $-4 a^{3}-27 b^{2}$.

\subsection{Raízes das equações cúbicas reduzidas com coeficientes complexos}

\subsubsection{O método de Cardano}

O método de Cardano para a determinação das raízes complexas da equação reduzida

$$
z^{3}+a z+b=0
$$

consiste em exprimir a incógnita $z$ como soma de duas parcelas $u$ e $v$, isto é,

$$
z=u+v
$$

e impondo que

$$
(u+v)^{3}+a(u+v)+b=0
$$

vem

$$
\left(u^{3}+v^{3}+b\right)+(3 u v+a)(u+v)=0 .
$$

Os valores de $u$ e $v$ devem ser tais que

$$
u^{3}+v^{3}=b
$$




$$
u^{3} v^{3}=-\frac{a^{3}}{27}
$$

o que mostra que $u^{3}$ e $v^{3}$ são raízes da equação quadrática

$$
Z^{2}-b Z-\frac{a^{3}}{27}=0
$$

isto é,

$$
\begin{aligned}
& u^{3}=\frac{b+\sqrt{b^{2}+\frac{4 a^{3}}{27}}}{2} \\
& v^{3}=\frac{b-\sqrt{b^{2}+\frac{4 a^{3}}{27}}}{2}
\end{aligned}
$$

Pela definição do discriminante de equações cúbicas, equações cúbicas com coeficientes complexos e discriminante nulo admitem raízes não todas distintas entre si, isto é, existem no máximo dois números complexos distintos que são raízes da equação cúbica.

No caso de equações cúbicas com coeficientes reais, equações cúbicas com discriminante positivo admitem três raízes reais distintas, e equações cúbicas com discriminante negativo admite uma única raiz real e um par de raízes complexas conjugadas.

A fórmula de Cardano exibe as raízes da equação cúbica reduzida $z^{3}+$ $a z+b=0$ :

$$
\begin{aligned}
& z_{1}=u+v \\
& z_{2}=w u+w^{2} v \\
& z_{3}=w^{2} u+w v
\end{aligned}
$$

em que

$$
w=\frac{1}{2}(-1+i \sqrt{3})
$$

é uma raiz cúbica da unidade, isto é, $w^{3}=1$, e

$$
u=\sqrt[3]{\frac{b}{2}+\sqrt{\frac{b^{2}}{4}+\frac{a^{3}}{27}}} \text { e } v=\sqrt[3]{\frac{b}{2}-\sqrt{\frac{b^{2}}{4}+\frac{a^{3}}{27}}}
$$


As fórmulas de Cardano permitem o cálculo do discriminante da equação cúbica reduzida

$$
z^{3}+a z+b=0
$$

pois

$$
\begin{aligned}
\left(z_{1}-z_{2}\right) & \left(z_{1}-z_{3}\right)\left(z_{2}-z_{3}\right) \\
& =(1-\omega)\left(u-\omega^{2} v\right)\left(1-\omega^{2}\right)(u-\omega v) \omega(1-\omega)(u-v) \\
& =(u-v)(u-\omega v)\left(u-\omega^{2} v\right) 3 \sqrt{3} i \\
& =\left(u^{3}-v^{3}\right) 3 \sqrt{3} i
\end{aligned}
$$

Pela fatoração

$$
\left(\frac{u}{v}\right)^{3}-1=\left(\frac{u}{v}-1\right)\left(\frac{u}{v}-\omega\right)\left(\frac{u}{v}-\omega^{2}\right)
$$

o discriminante $D$ da equação cúbica reduzida (e da equação cúbica completa), o qual por definição é

$$
D=\left(z_{1}-z_{2}\right)^{2}\left(z_{1}-z_{3}\right)^{2}\left(z_{2}-z_{3}\right)^{2}=\left(b^{2}+\frac{4 a^{3}}{27}\right) 27 i^{2}=-4 a^{3}-27 b^{2}
$$

\subsubsection{O método de Viète}

O método de Viète para a determinação das raízes complexas da equação cúbica reduzida com coeficientes complexos

$$
z^{3}+a z+b=0
$$

consiste na mudança de variável

$$
z=\frac{a}{3 w}-w
$$

e, por substituição,

$$
\begin{gathered}
\left(\frac{a}{3 w}-w\right)^{3}+a\left(\frac{a}{3 w}-w\right)+b=0 \\
w^{6}-b w^{3}-\left(\frac{a}{3}\right)^{3}=0
\end{gathered}
$$


que é uma equação quadrática em $w^{3}$, isto é,

$$
w^{3}=\frac{b}{2} \pm \frac{\sqrt{b^{2}+4\left(\frac{a}{3}\right)^{3}}}{2}=\frac{b}{2} \pm \sqrt{\left(\frac{a}{3}\right)^{3}+\left(\frac{b}{2}\right)^{2}}
$$

A escolha de $w_{1}^{3}=\frac{b}{2}+\sqrt{\left(\frac{a}{3}\right)^{3}+\left(\frac{b}{2}\right)^{2}}$ ou de $w_{2}^{3}=\frac{b}{2}-\sqrt{\left(\frac{a}{3}\right)^{3}+\left(\frac{b}{2}\right)^{2}}$ conduz ao mesmo valor de $z$, pois

$$
\left(w_{1} w_{2}\right)^{3}=-\left(\frac{a}{3}\right)^{3}
$$

$\mathrm{Ou}$

$$
w_{1} w_{2}=-\frac{a}{3}
$$

e

$$
-w_{1}+\frac{a}{3 w_{1}}=-w_{2}+\frac{a}{3 w_{2}}=-\frac{a}{3 w_{1}}-\frac{a}{\frac{-a}{w_{1}}}
$$

A fórmula de Cardano é obtida pelo método de Viète: de $z=w_{1}-\frac{a}{3 w_{1}}=$ $w_{1}+w_{2}$.

$$
z=\sqrt[3]{\frac{b}{2}+\sqrt{\left(\frac{a}{3}\right)^{3}+\left(\frac{b}{2}\right)^{2}}}+\sqrt[3]{\frac{b}{2}-\sqrt{\left(\frac{a}{3}\right)^{3}+\left(\frac{b}{2}\right)^{2}}}
$$

\subsubsection{O método de Gregory}

O método de Gregory para a determinação das raízes complexas da equação cúbica reduzida com coeficientes complexas

$$
z^{3}+a z+b=0
$$

consiste na expressão de $z$ como soma de duas parcelas $u$ e $v$, isto é,

$$
z=u+v
$$

a fim de obter duas equações quadráticas em $u^{3}$ e $v^{3}$, como

$$
\begin{gathered}
(u+v)^{3}+a(u+v)+b=0 \\
v^{3}+3 u v^{2}+\left(3 u^{2}+a\right) v+u^{3}+a u+b=0
\end{gathered}
$$

e como para quaisquer valores de $\alpha, \beta$ e $\gamma$,

$$
\left[v^{3}+\alpha v^{2}+\beta v+\gamma\right]\left[v^{3}+3 u v^{2}+\left(3 u^{2}+a\right) v+u^{3}+a u+b\right]=0
$$


O método de Gregory obtém a equação quadrática em $u^{3}$ :

$$
u^{6}-b u^{3}-\left(\frac{a^{3}}{3}\right)=0
$$

em que o primeiro membro da equação acima é o coeficiente de $v$ quando $\alpha, \beta$ e $\gamma$ são escolhidos de modo a anular os coeficientes de $v^{2}$, de $v^{4}$ e de $v^{5}$ na última equação:

o coeficiente de $v^{2}$ :

$$
\gamma(3 u)+\beta\left(3 u^{2}+a\right)+\alpha\left(u^{3}+a u+b\right)=0
$$

o coeficiente de $v^{4}$ :

$$
3 u^{2}+a+3 \alpha u+\beta=0
$$

o coeficiente de $v^{5}$ :

$$
3 u+\alpha=0
$$

o que implica

$$
\begin{gathered}
\alpha=-3 u \\
\beta=6 u^{2}-a \\
\gamma=\frac{a^{2}+3 b u-15 u^{4}}{3 u}
\end{gathered}
$$

Determinado $u$, o valor de $v$ é obtido pela equação quadrática em $v^{3}$,

$$
v^{6}+\left[\gamma+3 \beta u+\alpha\left(3 u^{2}+a\right)+u^{3}+a u+b\right] v^{3}+\gamma\left(u^{3}+a u+b\right)=0
$$

\subsection{Raízes das equações polinomiais quárticas: o método de Ferrari}

O método de Ferrari para a determinação das raízes complexas da equação quártica completa com coeficientes complexos

$$
Z^{4}+A Z^{3}+B Z^{2}+C Z+D=0
$$

utiliza, em primeiro lugar, a mudança de variável $z=Z+\frac{A}{4}$ para a obtenção da equação quártica reduzida

$$
z^{4}+a z^{2}+b z+c=0
$$


em que

$$
\begin{gathered}
a=B-6\left(\frac{A}{4}\right)^{2} \\
b=C-\frac{A}{2} B+\left(\frac{A}{2}\right)^{3} \\
c=D-\frac{A}{4} C+\left(\frac{A}{4}\right)^{2} B-3\left(\frac{A^{4}}{4}\right)
\end{gathered}
$$

e, em seguida, são utilizados dois completamentos de quadrados: o primeiro

$$
\left(z^{2}+\frac{a}{2}\right)^{2}=-b z-c+\left(\frac{a}{2}\right)^{2}
$$

e o segundo

$$
\left(z^{2}+\frac{a}{2}+t\right)^{2}=-b z-c+\left(\frac{a}{2}\right)^{2}+2 t z^{2}+a t+t^{2}
$$

e o objetivo está na determinação do valor de $t$ de modo que o segundo membro é expresso como um quadrado perfeito, isto é, quando $t \neq 0$,

$$
-b z-c+\left(\frac{a}{2}\right)^{2}+2 t z^{2}+a t+t^{2}=\left(\sqrt{2 t} z-\frac{b}{2 \sqrt{2 t}}\right)^{2}
$$

e então o discriminante da equação quadrática em z deve ser nulo, e

$$
8 t^{3}+8 a t^{2}+\left(2 a^{2}-8 c\right) t-b^{2}=0
$$

que é também denominada equação cúbica resolvente associada a equação quártica dada.

Obtido um valor não nulo de $t$, raiz da equação cúbica resolvente,

$$
z^{2}+\frac{a}{2}+t=\sqrt{2 t} z-\frac{b}{2 \sqrt{2 t}}
$$

$\mathrm{ou}$

$$
z^{2}+\frac{a}{2}+t=-\sqrt{2 t} z+\frac{b}{2 \sqrt{2 t}}
$$

as quais são duas equações quadráticas em $z$.

Quando $t=0, b=0$ e a equação quártica reduzida é uma equação quadrática em $z^{2}$

$$
z^{4}+a z^{2}+c=0
$$


cujas raízes são facilmente determinadas.

A equação polinomial quártica completa de coeficientes complexos

$$
Z^{4}+A Z^{3}+B Z^{2}+C Z+D=0
$$

e a equação quártica reduzida

$$
z^{4}+a z^{2}+b z+c=0
$$

obtida pela mudança de variável canônica

$$
Z=z-\frac{A}{4}
$$

admitem o mesmo discriminante $D$ dado pela fórmula

$$
D=-16 a^{4} c+4 a^{3} b^{2}+128 a^{2} c^{2}-144 a b^{2} c+27 b^{4}-256 c^{3}
$$

A primeira equação resolvente cúbica associada a equação quártica reduzida é a equação cúbica

$$
Z^{3}+2 a Z^{2}+\left(a^{2}-4 c\right) Z-b^{2}=0
$$

cujas raízes são $\left(z_{1}+z_{2}\right)\left(z_{3}+z_{4}\right),\left(z_{1}+z_{3}\right)\left(z_{2}+z_{4}\right),\left(z_{1}+z_{4}\right)\left(z_{2}+z_{3}\right)$ em que $z_{1}, z_{2}, z_{3}$ e $z_{4}$ são as raízes da equação quártica reduzida porque, devido as relações de Girard,

$$
\begin{gathered}
z_{1}+z_{2}+z_{3}+z_{4}=0 \\
z_{1} z_{2}+z_{1} z_{3}+z_{1} z_{4}+z_{2} z_{3}+z_{2}+z_{4}+z_{3} z_{4}=a \\
z_{1} z_{2} z_{3}+z_{1} z_{2} z_{4}+z_{1} z_{3} z_{4}+z_{2} z_{3} z_{4}=-b \\
z_{1} z_{2} z_{3} z_{4}=c
\end{gathered}
$$

a soma

$$
\left(z_{1}+z_{2}\right)\left(z_{3}+z_{4}\right)+\left(z_{1}+z_{3}\right)\left(z_{2}+z_{4}\right)+\left(z_{1}+z_{4}\right)\left(z_{2}+z_{3}\right)=-2 a
$$

a soma de todos os produtos tomados dois a dois é igual a $a^{2}-4 c$ e o produto

$$
\left(z_{1}+z_{2}\right)\left(z_{1}+z_{3}\right)\left(z_{1}+z_{4}\right)\left(z_{2}+z_{3}\right)\left(z_{2}+z_{4}\right)\left(z_{3}+z_{4}\right)=b^{2}
$$

Determinadas as raízes da equação cúbica resolvente

$$
\left(z_{1}+z_{2}\right)^{2}=-\left(z_{1}+z_{2}\right)\left(z_{3}+z_{4}\right)
$$




$$
\begin{aligned}
& \left(z_{1}+z_{3}\right)^{2}=-\left(z_{1}+z_{3}\right)\left(z_{2}+z_{4}\right) \\
& \left(z_{1}+z_{4}\right)^{2}=-\left(z_{1}+z_{4}\right)\left(z_{2}+z_{3}\right) \\
z_{1}= & \frac{1}{2}\left[\left(z_{1}+z_{2}\right)+\left(z_{1}+z_{3}\right)+\left(z_{1}+z_{4}\right)\right] \\
z_{2}= & \frac{1}{2}\left[\left(z_{1}+z_{2}\right)-\left(z_{1}+z_{3}\right)-\left(z_{1}+z_{4}\right)\right] \\
z_{3}= & \frac{1}{2}\left[-\left(z_{1}+z_{2}\right)+\left(z_{1}+z_{3}\right)-\left(z_{1}+z_{4}\right)\right] \\
z_{4}= & \frac{1}{2}\left[-\left(z_{1}+z_{2}\right)-\left(z_{1}+z_{3}\right)+\left(z_{1}+z_{4}\right)\right]
\end{aligned}
$$

A segunda equação cúbica resolvente associada a equação quártica reduzida

$$
z_{4}+a z^{2}+b z+c=0
$$

é definida por

$$
Z^{3}-a Z^{2}-4 c Z+4 a c-b^{2}=0
$$

cujas raízes são

$$
z_{1} z_{2}+z_{3} z_{4}, z_{1} z_{3}+z_{2} z_{4}, z_{1} z_{4}+z_{2} z_{3}
$$

em que $z_{1}, z_{2}, z_{3}$ e $z_{4}$ são as raízes da equação quártica reduzida porque devido às relações de Girard,

$$
\begin{gathered}
\left(z_{1} z_{2}+z_{3} z_{4}\right)+\left(z_{1} z_{3}+z_{2} z_{4}\right)+\left(z_{1} z_{4}+z_{2} z_{3}\right)=a \\
\left(z_{1} z_{2}+z_{3} z_{4}\right)\left(z_{1} z_{3}+z_{2} z_{4}\right)\left(z_{1} z_{4}+z_{2} z_{3}\right)=b^{2}-4 a c
\end{gathered}
$$

e a soma de todos os produtos tomados dois a dois é igual a $-4 c$ e, neste caso, o fato de que as raízes de uma equação quártica são distintas entre si implica que as raízes da equação cúbica resolvente também são distintas

$$
z_{1} z_{2}+z_{3} z_{4}-\left(z_{1} z_{3}+z_{2} z_{4}\right)=\left(z_{1}-z_{4}\right)\left(z_{2}-z_{4}\right)
$$

o que implica, além disso, que os discriminantes da equação quadrática e da equação cúbica resolvente são iguais.

Determinadas as raízes $w_{1}, w_{2}$ e $w_{3}$ da equação cúbica resolvente

$$
\begin{aligned}
& w_{1}=z_{1} z_{2}+z_{3} z_{4} \\
& w_{2}=z_{1} z_{3}+z_{2} z_{4} \\
& w_{3}=z_{1} z_{4}+z_{2} z_{3}
\end{aligned}
$$


então

$$
\begin{gathered}
\left(z_{1}+z_{3}\right)\left(z_{2}+z_{4}\right)=w_{1} w_{3} \\
\left(z_{1}+z_{2}\right)+\left(z_{3}+z_{4}\right)=0
\end{gathered}
$$

o que mostra que

$$
\begin{aligned}
& z_{1}+z_{3}=\sqrt{-w_{1}-w_{3}} \\
& z_{2}+z_{4}=\sqrt{-w_{1}-w_{3}}
\end{aligned}
$$

e que

$$
\begin{aligned}
& z_{1}+z_{3}=\sqrt{w_{2}-a} \\
& z_{2}+z_{4}=\sqrt{w_{2}-a}
\end{aligned}
$$

analogamente,

$$
\begin{aligned}
z_{1}+z_{4} & =\sqrt{w_{3}-a} \\
z_{2}+z_{3} & =\sqrt{w_{1}-a} \\
z_{1}+z_{2} & =\sqrt{w_{1}-a} \\
z_{3}+z_{4} & =-\sqrt{w_{1}-a}
\end{aligned}
$$

para finalmente chegar às fórmulas de Ferrari

$$
\begin{aligned}
& 2 z_{1}=\sqrt{w_{1}-a}+\sqrt{w_{2}-a}+\sqrt{w_{3}-a} \\
& 2 z_{2}=\sqrt{w_{1}-a}-\sqrt{w_{2}-a}-\sqrt{w_{3}-a} \\
& 2 z_{3}=-\sqrt{w_{1}-a}+\sqrt{w_{2}-a}-\sqrt{w_{3}-a} \\
& 2 z_{4}=-\sqrt{w_{1}-a}-\sqrt{w_{2}-a}+\sqrt{w_{3}-a}
\end{aligned}
$$

\subsection{Raízes das equações quárticas reduzidas com coeficientes complexos}

\subsubsection{O método de Cartan-Ferrari}

O Método de Cartan-Ferrari para a determinação das raízes da equação quártica reduzida com coeficientes complexos

$$
z^{4}+a z^{2}+b z+c=0
$$


consiste na expressão de $z^{2}$ como diferença de duas parcelas $u$ e $v$, isto é,

$$
z^{2}=u-v
$$

$\log \mathrm{O}$

$u^{2}=\left(z^{2}+v\right)^{2}=z^{4}+v^{2}+2 v z^{2}=-a z^{2}-b z-c+v^{2}+2 v z^{2}=(2 v-a) z^{2}-b z+\left(v^{2}-c\right)$

e a última expressão iguala

$$
u^{2}=(\alpha z+\beta)^{2}
$$

desde que $v$ é raiz do discriminante da equação quadrática

$$
\begin{gathered}
b^{2}-4(2 v-a)\left(v^{2}-c\right)=0 \\
\text { ou } 8 v^{3}-4 a v^{2}-8 c v-\left(b^{2}-4 a c\right)=0
\end{gathered}
$$

a qual é uma equação cúbica resolvente associada a equação quártica.

Determinados $\alpha$ e $\beta$ pelas equações

$$
\begin{gathered}
\alpha^{2}=2 v-a \\
\beta^{2}=v^{2}-c
\end{gathered}
$$

cujos sinais são compatíveis com

$$
\begin{gathered}
2 \alpha \beta=-b \\
\text { obtem-se }\left(z^{2}+v\right)^{2}=(\alpha z+\beta)^{2}=0 \\
\text { ou }\left(z^{2}+v+\alpha z+\beta\right)\left(z^{2}+v-\alpha z-\beta\right)=0 .
\end{gathered}
$$

As raízes da equação quártica são as raízes de duas equações quadráticas e o caso em que $v=\frac{a}{2}$ é o caso da equação biquadrada

$$
\left[z^{2}+\frac{a}{2}\right]^{2}=\left(\frac{a}{2}\right)^{2}-c
$$

\subsubsection{O método de Gregory}

O Método de Gregory para a determinação das raízes da equação quártica reduzida com coeficientes complexos

$$
z^{4}+a z^{2}+b z+c=0
$$


consiste na expressão de $z$ como soma de duas parcelas $u$ e $v$ a fim de obter duas equações cúbicas em $u^{3}$ e $v^{3}$; como

$$
\begin{gathered}
\text { e }(u+v)^{4}+a(u+v)^{2}+b(u+v)+c=0 \\
v^{4}+4 u v^{3}+\left(6 u^{2}+a\right) v^{2}+\left(4 u^{3}+2 a u+b\right) v+u^{4}+a u^{2}+b u+c=0
\end{gathered}
$$

para quaisquer valores de $\alpha$ e $\beta$

$\left[v^{2}+\alpha v+\beta\right]\left[v^{4}+4 u v^{3}+\left(6 u^{2}+a\right) v^{2}+\left(4 u^{3}+2 a u+b\right) v+u^{4}+a u^{2}+b u+c\right]=0$

o método de Gregory obtém a equação cúbica em $u^{2}$.

$$
64 u^{6}+32 a u^{4}+\left(4 a^{2}-16 c\right) u^{2}-b^{2}=0
$$

quando $\alpha$ e $\beta$ são escolhidos de modo a obter o anulamento do coeficiente de $v^{3}$ e de $v^{5}$ na última equação:

coeficiente de $v^{3}$

$$
4 u^{3}+2 a u+b+\alpha\left(6 u^{2}+a\right)+4 \beta u=0
$$

coeficiente de $v^{5}$

$$
4 u+\alpha=0
$$

o que implica

$$
\begin{gathered}
\alpha=-4 u \\
4 \beta u=-4 u^{3}-2 a u-b+4 u\left(6 u^{2}+a\right)
\end{gathered}
$$

que, substituídos na equação do coeficiente de $v$

$$
\alpha\left(u^{4}+a u^{2}+b u+c\right)+\beta\left(4 u^{3}+2 a u+b\right)=0
$$

conduz a equação cúbica em $u^{2}$.

\subsubsection{O método de Tschinhaus}

Dada a equação cúbica completa mônica com coeficientes complexos

$$
P(Z)=Z^{3}+A Z^{2}+B Z+C=0
$$

e definindo os polinômios derivados de $P$ por

$$
P^{\prime}(Z)=3 Z^{2}+2 A Z+B
$$




$$
\begin{gathered}
P^{\prime \prime}(Z)=6 Z+2 A \\
P^{\prime \prime \prime}(Z)=6
\end{gathered}
$$

a fórmula de Taylor

$$
P(Z)=P\left(Z_{0}\right)+P^{\prime}\left(Z_{0}\right)\left(Z-Z_{0}\right)+P^{\prime \prime}\left(Z_{0}\right)\left(Z-Z_{0}\right)^{2}+P^{\prime \prime \prime}\left(Z_{0}\right)\left(Z-Z_{0}\right)^{3}
$$

mostra que

1. quando $Z_{0} \in \mathbb{C}$ é tal que $P^{\prime \prime}\left(Z_{0}\right)=6 Z_{0}+2 A=0$ a mudança de variável $z=Z-Z_{0}$ transforma a equação cúbica dada em uma equação cúbica reduzida em que não ocorre o termo em $z^{2}$

2. quando $Z_{0} \in \mathbb{C}$ e $P^{\prime}\left(Z_{0}\right)=3 Z_{0}^{2}+2 A Z_{0}+B=0$ a equação cúbica em uma equação reduzida em que não ocorre o termo em $z$.

O método de TSCHINHAUS aplicado a equações cúbicas transforma a equação cúbica com coeficientes complexos

$$
P(z)=z^{3}+a z+b=0
$$

na equação cúbica incompleta

$$
w^{3}=\gamma^{3}
$$

com uma mudança de variável da forma $w=z^{2}+\alpha z+\beta$.

Para a determinação dos valores de $\alpha, \beta$ e $\gamma$ resolvendo

$$
z^{2}+\alpha z+\beta-w=0
$$

para obter

$$
\begin{aligned}
& z_{1}=\frac{-\alpha+\sqrt{\alpha^{2}+4(w-\beta)}}{2} \\
& z_{2}=\frac{-\alpha-\sqrt{\alpha^{2}+4(w-\beta)}}{2}
\end{aligned}
$$

e calculando

$$
\left(z_{1}^{3}+a z_{1}+b\right)\left(z_{2}^{3}+a z_{2}+b\right)=0
$$

obtém-se

$-w^{3}+(3 \beta-2 a) w^{2}+\left(4 a \beta-a \alpha^{2}-3 \beta^{2}-a^{2}-3 \alpha b\right) w+\left(\beta^{3}-2 a \beta^{2}-a b \alpha+a^{2} \beta+b^{2}-b \alpha^{3}+3 b \alpha \beta=0\right.$ 
Definindo

$$
D=\sqrt{\left(\frac{a}{3}\right)^{2}+\left(\frac{b}{2}\right)^{2}}
$$

e escolhido

$$
\begin{gathered}
\alpha=\frac{3}{a}\left[\frac{-b}{2}+D\right] \\
\beta=\frac{2 a}{3}
\end{gathered}
$$

a equação para $w$ fica

$$
w^{3}=\left[\frac{6 D}{a} \gamma\right]^{3}
$$

em que

$$
\gamma^{3}=D-\frac{b}{2}
$$

O resto $R(z)$ da divisão euclidiana de $z^{3}+a z+b$ por $z^{2}+\alpha z+(\beta-w)$ é igual a

$$
R(z)=\left(a-\beta+b^{2}+w\right) z+b+\alpha \beta-\alpha w
$$

e a menos de uma constante real não nula é da forma

$$
z-\gamma+\frac{a}{3 \gamma}
$$

que, igualado a zero, recai na fórmula de Cardano

$$
z=\gamma-\frac{a}{2 \gamma} .
$$




\section{Capítulo 2}

\section{Equações Polinomiais com coeficientes complexos}

\subsection{Teorema da Limitação das Raízes complexas}

Teorema 2.1 (Teorema da Limitação das Raízes complexas). Seja z uma raiz complexa da equação polinomial com coeficientes complexos de grau $N \geqslant 1$,

$$
P(Z)=Z^{N}+a_{N-1} Z^{N-1}+\cdots+a_{1} Z+a_{0}=0, \quad a_{0}, a_{1}, \ldots, a_{N-1} \in \mathbb{C} .
$$

Então

(A) $|z| \leqslant 1+\left|a_{0}\right|+\left|a_{1}\right|+\cdots+\left|a_{N-1}\right|$ ou $|z| \leqslant \max \left\{1,\left|a_{0}\right|+\left|a_{1}\right|+\cdots+\left|a_{N-1}\right|\right\}$.

(B) $|z| \leqslant \frac{1}{\sqrt[N]{2}-1} \max \left\{\left[\frac{\left|a_{N-K}\right|}{\left(\begin{array}{l}N \\ K\end{array}\right)}\right]^{1 / K}: K=1,2, \ldots, N\right\}$.

(C) $|z| \leqslant 2 \max \left\{\left|a_{N-K}\right|^{1 / K}: K=1,2, \ldots, N\right\}$.

(D) $|z| \leqslant\left|a_{N-1}\right|+\max \left\{\left(\frac{\left|a_{N-K}\right|}{\left|a_{N-1}\right|}\right)^{\frac{1}{K-1}}: K=1,2, \ldots, N\right\}$.

(E) $|z| \leqslant R$, onde $R$ é a única raiz real positiva da equação polinomial

$$
x^{N}-b_{N-1} x^{N-1}-\cdots-b_{1} x-b_{0}=0
$$

com coeficientes reais de grau $N \geqslant 1$ em que

$$
b_{0} \geqslant\left|a_{0}\right|, \quad b_{1} \geqslant\left|a_{1}\right|, \ldots, \quad b_{N-1} \geqslant\left|a_{N-1}\right| .
$$


No caso em que

$$
P(Z)=Z^{N}+a_{N-r} Z^{N-r}+\cdots+a_{1} Z+a_{0} \neq 0,
$$

(F) $|z| \leqslant 1+\sqrt[r]{\max \left\{\left|a_{K}\right|: K=0,1,2, \ldots, N-r\right\}}$.

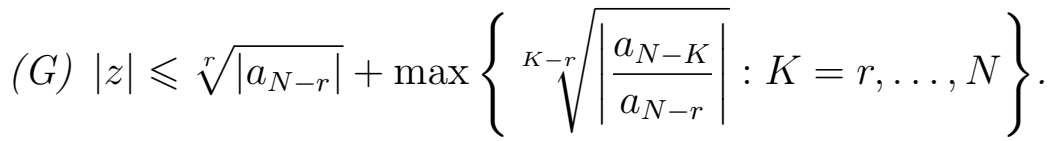

Demonstração de $(B)-H$. Zassenhaus. Seja $M$ um número real positivo tal que

$$
M \geqslant\left[\frac{\left|a_{N-K}\right|}{\left(\begin{array}{l}
N \\
K
\end{array}\right)}\right]^{1 / k}, \quad K=1,2, \ldots, N
$$

isto é,

$$
\left|a_{N-K}\right| \leqslant\left(\begin{array}{l}
N \\
K
\end{array}\right) M^{K} \quad \text { para } K=1,2, \ldots N .
$$

Quando $P(Z)=0$,

$$
\begin{aligned}
|Z|^{N} & =\left|a_{N-1} Z^{N-1}+\cdots+a_{1} Z+a_{0}\right| \\
& \leqslant\left|a_{N-1}\right||Z|^{N-1}+\cdots+\left|a_{1}\right||Z|+\left|a_{0}\right| \\
& \leqslant\left(\begin{array}{c}
N \\
1
\end{array}\right) M|Z|^{N-1}+\cdots+\left(\begin{array}{c}
N \\
N-1
\end{array}\right) M^{N-1}|Z|+M^{N} \\
& =(|Z|+M)^{N}-|Z|^{N}
\end{aligned}
$$

Então

$$
2|Z|^{N} \leqslant(|Z|+M)^{N} \Longrightarrow(\sqrt[N]{2}-1)|Z| \leqslant M
$$

e portanto

$$
|Z| \leqslant \frac{M}{\sqrt[N]{2}-1}
$$

De 2.4 para o caso particular

$$
M=\max \left\{\left[\frac{\left|a_{N-K}\right|}{\left(\begin{array}{l}
N \\
K
\end{array}\right)}\right]^{1 / K}: K=1,2, \ldots, N\right\}
$$

segue que

$$
|z| \leqslant \frac{1}{\sqrt[N]{2}-1} \max \left\{\left[\frac{\left|a_{N-K}\right|}{\left(\begin{array}{l}
N \\
K
\end{array}\right)}\right]^{1 / K}: K=1,2, \ldots, N\right\} .
$$


Demonstração de (C). Seja

$$
M=\max \left\{\left|a_{N-K}\right|^{1 / K}: K=1,2, \ldots, N\right\} .
$$

Então

$$
\left|a_{N-K}\right| \leqslant M^{K}, \quad K=1,2, \ldots N .
$$

Quando $P(Z)=0$ e levando em conta 2.5 , temos

$$
\begin{aligned}
|Z|^{N} & \leqslant\left|a_{N-1}\right||Z|^{N-1}+\cdots+\left|a_{1}\right||Z|+\left|a_{0}\right| \\
& \leqslant M|Z|^{N-1}+\cdots+M^{N-1}|Z|+M^{N} \\
\left|\frac{Z}{M}\right|^{N} & \leqslant\left(\frac{|Z|}{M}\right)^{N-1}+\cdots+\left(\frac{|Z|}{M}\right)+1 \\
& =\frac{\left|\frac{Z}{M}\right|^{N}-1}{\left|\frac{Z}{M}\right|-1} .
\end{aligned}
$$

Se $\left|\frac{Z}{M}\right| \geqslant 2$, então o denominador em 2.9 satisfaz $\left|\frac{Z}{M}\right|-1 \geqslant 1$ e tiramos de (2.9) que

$$
\left|\frac{Z}{M}\right|^{N} \leqslant\left|\frac{Z}{M}\right|^{N}-1,
$$

isto é, $0 \leqslant-1$, uma contradição.

Conclusão: Se $P(Z)=0$ então $|Z| \leqslant 2 M$, ou seja,

$$
|z| \leqslant 2 \max \left\{\left|a_{N-K}\right|^{1 / K}: K=1,2, \ldots, N\right\} .
$$

Exemplo 2.2. Dado

$$
P(Z)=Z^{5}+17 Z^{4}-5 Z^{3}-277 Z^{2}+144,
$$

os módulos das raízes complexas de $P(Z)=0$ não ultrapassam

$$
\begin{gathered}
1+17+5+277+144=444, \\
\frac{1}{\sqrt[5]{2}-1} \max \left\{\frac{17}{5}, \frac{1}{\sqrt{2}}, \sqrt[3]{\frac{277}{10}}, \sqrt[4]{0}, \sqrt[5]{144}\right\} \leqslant 23, \\
2 \max \{17, \sqrt{5}, \sqrt[3]{277}, \sqrt[4]{0}, \sqrt[5]{144}\}=34 \\
17+\max \left\{1, \frac{5}{17}, \sqrt{\frac{277}{17}}, \sqrt[3]{0}, \sqrt[4]{\frac{144}{17}}\right\}<22 .
\end{gathered}
$$


Exemplo 2.3. Dado

$$
P(Z)=Z^{5}+2 Z^{4}-8 Z^{3}+2 Z^{2}+3 Z+7
$$

os módulos das raízes complexas de $P(Z)=0$ não ultrapassam:

$$
\begin{gathered}
1+2+8+2+3+7=23 \\
\frac{1}{\sqrt[5]{2}-1} \max \left\{\frac{2}{5}, \sqrt{\frac{4}{5}}, \sqrt[3]{\frac{1}{5}}, \sqrt[4]{\frac{1}{3}}, \sqrt[5]{7}\right\}<10 \\
2 \max \{17, \sqrt{8}, \sqrt[3]{2}, \sqrt[4]{3}, \sqrt[5]{7}\}=4 \sqrt{2}<6 .
\end{gathered}
$$

\subsection{Teorema Fundamental da Álgebra}

O teorema fundamental da álgebra estabelece que o conjunto $\mathbb{C}$ de todos os números complexos, com as operações usuais de adição e de multiplicação, constitui um corpo algebricamente fechado.

O significado deste enunciado é que toda equação polinomial de grau $N$ com coeficientes complexos da forma

$$
P(Z)=A_{N} Z^{N}+A_{N-1} Z^{N-1}+\cdots+A_{1} Z+A_{0}=0
$$

com $A_{0}, A_{1}, \ldots, A_{N-1}$ e $A_{N} \neq 0$ admite $N$ raízes complexas $Z_{1}, Z_{2}, \ldots, Z_{N}$ não necessariamente distintas, isto é, para cada $j=1,2, \ldots, N$, temos $P\left(Z_{j}\right)=$ 0 .

O que é equivalente a afirmar que toda função polinomial $P$ de grau $N$ com coeficientes complexos admite fatoração como produto de $N$ fatores lineares não necessariamente distintos:

$$
P(Z)=A_{N}\left(Z-Z_{1}\right)\left(Z-Z_{2}\right) \ldots\left(Z-Z_{N}\right) .
$$

A igualdade entre os coeficientes das expressões de $P(Z)$ em 2.10 e 2.11 acarreta as relações de GIRARD envolvendo as raízes $Z_{1}, Z_{2}, \ldots, Z_{N}$ das duas expressões acima de $P(Z)$ e os coeficientes $A_{0}, A_{1}, \ldots, A_{N}$.

\subsection{Relações de Girard}

$$
Z_{1}+Z_{2}+\cdots+Z_{N}=-\frac{A_{N-1}}{A_{N}}
$$




$$
\begin{gathered}
Z_{1} Z_{2}+Z_{1} Z_{3}+\cdots+Z_{1} Z_{N}+\cdots+Z_{2} Z_{3}+\cdots+Z_{2} Z_{N}=\frac{A_{N-2}}{A_{N}} \\
Z_{1} Z_{2} \ldots Z_{N}=(-1)^{N} \frac{A_{0}}{A_{N}}
\end{gathered}
$$

Definição 2.4 (Função polinomial derivada $\boldsymbol{P}^{\prime}$ da função polinomial $\boldsymbol{P}$ ). Se

$$
P(Z)=A_{N} Z^{N}+A_{N-1} Z^{N-1}+\cdots+A_{1} Z+A_{0}
$$

então

$$
P^{\prime}(Z)=N A_{N} Z^{N-1}+(N-1) A_{N-1} Z^{N-2}+\cdots+A_{1} .
$$

Podemos tomar derivadas sucessivas do polinômio $P$, obtendo $P^{\prime}, P^{\prime \prime}, P^{\prime \prime \prime}$, $P^{(4)}$, etc.

Definição 2.5 (Multiplicidade de raízes). Uma raiz $Z_{j}$ da equação polinomial $P(Z)=0$ é uma

1. raiz simples de $P(Z)=0$ quando

$$
P(Z)=\left(Z-Z_{j}\right) Q(Z) \quad \text { com } \quad Q\left(Z_{j}\right) \neq 0
$$

ou equivalentemente,

$$
P\left(Z_{j}\right)=0, \quad P^{\prime}\left(Z_{j}\right) \neq 0 .
$$

2. raiz dupla de $P(Z)=0$ quando

$$
P(Z)=\left(Z-Z_{j}\right)^{2} Q(Z) \quad \text { com } \quad Q\left(Z_{j}\right) \neq 0,
$$

ou equivalentemente,

$$
P\left(Z_{j}\right)=0, \quad P^{\prime}\left(Z_{j}\right)=0, \quad P^{\prime \prime}\left(Z_{j}\right) \neq 0 .
$$

3. raiz de multiplicidade $m_{j}$ de $P(Z)=0$ quando

$$
P(Z)=\left(Z-Z_{j}\right)^{m_{j}} Q(Z) \quad \text { com } \quad Q\left(Z_{j}\right) \neq 0,
$$

ou equivalentemente,

$$
\begin{gathered}
P\left(Z_{j}\right)=P^{\prime}\left(Z_{j}\right)=\cdots=P^{\left(m_{j}-1\right)}\left(Z_{j}\right)=0, \\
P^{m_{j}}\left(Z_{j}\right) \neq 0 .
\end{gathered}
$$


Sejam $Z_{1}, Z_{2}, \ldots, Z_{s}$ raízes distintas da equação polinomial $P(Z)=0$ com multiplicidades $m_{1}, m_{2}, \ldots, m_{s}$ respectivamente. Então:

$$
m_{1}+m_{2}+\cdots+m_{s}=N
$$

e

$$
P(Z)=A_{N}\left(Z-Z_{1}\right)^{m_{1}}\left(Z-Z_{2}\right)^{m_{2}} \ldots\left(Z-Z_{0}\right)^{m_{s}}
$$

O teorema de TAYLOR para funções polinomiais afirma que, quando $Z \in \mathbb{C}$ e $H \in \mathbb{C}$,

$P(Z+H)=P(Z)+P^{\prime}(Z) H+P^{\prime \prime}(Z) \frac{H^{2}}{2 !}+P^{\prime \prime \prime}(Z) \frac{H^{3}}{3 !}+\cdots+\cdots+P^{N}(Z) \frac{H^{N}}{N !}$

$\operatorname{mas}$ para $Z \neq Z_{1}, Z \neq Z_{2}, \ldots, Z \neq Z_{N}$

$$
\begin{aligned}
& P(Z+H) \\
& \quad=A_{N}\left(Z+H-Z_{1}\right)\left(Z+H-Z_{2}\right) \ldots\left(Z+H-Z_{N}\right) \\
& \quad=A_{N}\left(Z-Z_{1}\right)\left(Z-Z_{2}\right) \ldots\left(Z-Z_{N}\right)\left[1+\frac{H}{Z-Z_{1}}\right] \ldots\left[1+\frac{H}{Z-Z_{N}}\right] \\
& \quad=P(Z)\left[1+\frac{H}{Z-Z_{1}}\right] \ldots\left[1+\frac{H}{Z-Z_{N}}\right] .
\end{aligned}
$$

Igualando os coeficientes em $H$ nas duas expressões de $P(Z+H)$ temos, portanto, para $Z \neq Z_{1}, Z \neq Z_{2}, \ldots, Z \neq Z_{N}$

$$
\frac{P^{\prime}(Z)}{P(Z)}=\frac{1}{Z-Z_{1}}+\frac{1}{Z-Z_{2}}+\cdots+\frac{1}{Z-Z_{N}}
$$

e, no caso de $Z_{1}, Z_{2}, \ldots, Z_{s}$ serem raízes distintas da equação $P(Z)=0$ com multiplicidades $m_{1}, m_{2}, \ldots, m_{s}$, para $Z \neq Z_{1}, Z \neq Z_{2}, \ldots, Z \neq Z_{s}$, temos

$$
\frac{P^{\prime}(Z)}{P(Z)}=\frac{m_{1}}{Z-Z_{1}}+\frac{m_{2}}{Z-Z_{2}}+\cdots+\frac{m_{s}}{Z-Z_{s}}
$$

Assim, quando $Z_{1}, Z_{2}, \ldots, Z_{s}$ são as raízes distintas da equação $P(Z) \neq 0$,

$$
\begin{aligned}
& P(Z)=\left(Z-Z_{1}\right)^{m_{1}} P_{1}(Z) \\
& P(Z)=\left(Z-Z_{2}\right)^{m_{2}} P_{2}(Z)
\end{aligned}
$$




$$
P(Z)=\left(Z-Z_{s}\right)^{m_{s}} P_{s}(Z)
$$

o que mostra que

$$
\frac{P_{1}^{\prime}(Z)}{P_{1}(Z)}=\frac{m_{2}}{\left(Z-Z_{2}\right)^{m_{2}}}+\cdots+\frac{m_{s}}{\left(Z-Z_{s}\right)^{m_{s}}}
$$

e que

$$
\frac{P^{\prime}(Z)}{P(Z)}=\frac{m_{1}}{\left(Z-Z_{1}\right)^{m_{1}}}+\frac{P_{1}^{\prime}(Z)}{P_{1}(Z)}
$$

Analogamente,

$$
\begin{gathered}
\frac{P^{\prime}(Z)}{P(Z)}=\frac{m_{2}}{\left(Z-Z_{2}\right)^{m_{2}}}+\frac{P_{2}^{\prime}(Z)}{P_{2}(Z)} \\
\vdots \\
\frac{P^{\prime}(Z)}{P(Z)}=\frac{m_{s}}{\left(Z-Z_{s}\right)^{m_{s}}}+\frac{P_{s}^{\prime}(Z)}{P_{s}(Z)}
\end{gathered}
$$

que são fórmulas necessárias para a compreensão do Teorema de Rolle para funções polinomiais com coeficientes reais.

\subsection{Regra de Briot-Ruffini}

O dispositivo prático ou regra de Briot-Ruffini aplicado a uma equação polinomial com coeficientes complexos de grau $N \geqslant 1$

$$
P(Z)=A_{N} Z^{N}+A_{N-1} Z^{N-1}+\cdots+A_{1} Z+A_{0}
$$

em que $A_{0}, A_{1}, \ldots, A_{N-1}, A_{N} \in \mathbb{C}$, e $A_{N} \neq 0$ calcula os coeficientes do polinômio quociente $Q(Z)$ e o resto $\mathrm{R}$ resultantes da divisão euclidiana de $P(Z)$ por $\left(Z-Z_{0}\right)$, isto é,

$$
P(Z)=\left(Z-Z_{0}\right) \cdot Q(Z)+R
$$

em que $Q(Z)$ é um polinômio com coeficientes complexos de grau $N-1$

$$
Q(Z)=B_{N-1} Z^{N-1}+B_{N-2} Z^{N-2}+\cdots+B_{1} Z+B_{0}
$$

e os coeficientes $B_{N-1}, B_{N-2}, \ldots, B_{1}, B_{0}$ e $R$ são obtidos impondo a igualdade entre os coeficientes dos dois membros de

$$
P(Z)=A_{N} Z^{N}+A_{N-1} Z^{N-1}+\cdots+A_{1} Z+A_{0}
$$




$$
\begin{aligned}
& =\left(Z-Z_{0}\right) \cdot Q(Z)+R \\
& =\left(Z-Z_{0}\right) \cdot\left(B_{N-1} Z^{N-1}+B_{N-2} Z^{N-2}+\cdots+B_{1} Z+B_{0}\right)+R
\end{aligned}
$$

temos

$$
\begin{gathered}
A_{0}=-B_{0} Z_{0}+R \\
A_{1}=-B_{1} Z_{0}+B_{0} \\
A_{2}=-B_{2} Z_{0}+B_{1} \\
\quad \vdots \\
A_{N-2}=-B_{N-2} Z_{0}+B_{N-3} \\
A_{N-1}=-B_{N-1} Z_{0}+B_{N-2} \\
A_{N}=B_{N-1}
\end{gathered}
$$

$\mathrm{Ou}$

$$
\begin{aligned}
B_{N-1}= & A_{N} \\
B_{N-2}= & A_{N-1}+B_{N-1} Z_{0} \\
B_{N-3}= & A_{N-2}+B_{N-2} Z_{0} \\
& \vdots \\
B_{1}= & A_{2}+B_{2} Z_{0} \\
B_{0}= & A_{1}+B_{1} Z_{0} \\
R= & A_{0}+B_{0} Z_{0}
\end{aligned}
$$

e estas igualdades são mais rapidamente descritas com o dispositivo de BriotRuffini.

\begin{tabular}{c|l|l|l|l|l|l} 
& $A_{N}$ & $A_{N-1}$ & $\cdots$ & $A_{2}$ & $A_{1}$ & $A_{0}$ \\
\hline$Z_{0}$ & $\underbrace{A_{N}}_{=B_{N-1}}$ & $\underbrace{A_{N-1}+B_{N-1} \cdot Z_{0}}_{=B_{N-2}}$ & $\cdots$ & $\underbrace{A_{2}+B_{2} \cdot Z_{0}}_{=B_{1}}$ & $\underbrace{A_{1}+B_{1} \cdot Z_{0}}_{=B_{0}}$ & $\underbrace{A_{0}+B_{0} \cdot Z_{0}}_{=R}$
\end{tabular}.

Uma das aplicações do dispositivo prático de Briot-Ruffini é o cálculo rápido dos coeficientes

$$
P(H), P^{\prime}(H), \frac{P^{\prime \prime}(H)}{2 !}, \ldots, \frac{P^{(N)}(H)}{N !}
$$


da fórmula de Taylor

$$
P(Z+H)=P(H)+P^{\prime}(H) Z+\frac{P^{\prime \prime}(H) Z^{2}}{2 !}+\cdots+\frac{P^{(N)}(H) Z^{N}}{N !}
$$

em vista de que a divisão de $P(Z+H)$ por $Z$ resulta no resto constante $P(H)$ e no quociente

$$
P^{\prime}(H)+\frac{P^{\prime \prime}(H) Z}{2 !}+\cdots+\frac{P^{(N)}(H) Z^{N-1}}{N !}
$$

e a divisão deste quociente por $Z$ resulta no resto constante $P^{\prime}(H)$ e no quociente

$$
\frac{P^{\prime \prime}(H)}{2 !}+\cdots+\frac{P^{(N)}(H) Z^{N-2}}{N !}
$$

Exemplo 2.6. Para transformar a equação quártica com coeficientes inteiros

$$
P(Z)=Z^{4}-4 Z^{3}-18 Z^{2}-3 Z+2=0
$$

em uma equação quártica desprovida do termo de segundo grau através de uma mudança de variável de forma $Z=W+H$ considere a fórmula de Taylor

$$
P(W+H)=P(H)+P^{\prime}(H) W+\frac{P^{\prime \prime}(H) W^{2}}{2 !}+\frac{P^{\prime \prime \prime}(H) W^{3}}{3 !}+\frac{P^{\prime \prime \prime \prime}(H) W^{4}}{4 !}
$$

em que

$$
\begin{gathered}
P^{\prime}(H)=4 H^{3}-12 H^{2}-36 H-3 \\
P^{\prime \prime}(H)=12\left(H^{2}-2 H-3\right)=12(H+1)(H-3) \\
P^{\prime \prime \prime}(H)=24 H-24 \\
P^{\prime \prime \prime \prime}(H)=24
\end{gathered}
$$

e escolha $H=3$ raiz de $P^{\prime \prime}(H)=0$. O dispositivo de Briot-Ruffini, calcula

$$
P(3), \quad P^{\prime}(3), \quad \frac{P^{\prime \prime}(3)}{2 !}=0, \quad \frac{P^{\prime \prime \prime}(3)}{3 !}, \quad \frac{P^{\prime \prime \prime \prime}(3)}{4 !}
$$

\begin{tabular}{l|lllll} 
& 1 & -4 & -18 & -3 & 2 \\
\hline 3 & 1 & -1 & -21 & -66 & $-196=P(3)$ \\
3 & 1 & 2 & -15 & $-111=P^{\prime}(3)$ & \\
3 & 1 & 5 & $0=\frac{P^{\prime \prime}(3)}{2 !}$ & \\
3 & 1 & $8=\frac{P^{\prime \prime \prime}(3)}{3 !}$ & & \\
3 & $1=\frac{P^{\prime \prime \prime \prime}(3)}{4 !}$ & & &
\end{tabular}


Escolhido $H=-1$, o dispositivo de Briot-Ruffini calcula

$$
P(-1), \quad P^{\prime}(-1), \quad \frac{P^{\prime \prime}(-1)}{2 !}=0, \quad \frac{P^{\prime \prime \prime}(-1)}{3 !}, \quad \frac{P^{\prime \prime \prime \prime}(-1)}{4 !}
$$

\begin{tabular}{l|lllll} 
& 1 & -4 & -18 & -3 & 2 \\
\hline-1 & 1 & -5 & -13 & 10 & $-8=P(-1)$ \\
-1 & 1 & -6 & -7 & $17=P^{\prime}(-1)$ & \\
-1 & 1 & -7 & $0=\frac{P^{\prime \prime}(-1)}{2 !}$ & \\
-1 & 1 & $-8=\frac{P^{\prime \prime \prime}(-1)}{3 !}$ & & & \\
-1 & $1=\frac{P^{\prime \prime \prime \prime}(-1)}{4 !}$ & & & &
\end{tabular}

Portanto,

$$
\begin{gathered}
P(Z)=(Z-3)^{4}+8(Z-3)^{3}-111(Z-3)-196 \\
P(Z)=(Z+1)^{4}+8(Z+1)^{3}+17(Z+1)-8
\end{gathered}
$$

e a mudança de variável $W=Z-3$ transforma a equação quártica dada

$$
Z^{4}-4 Z^{3}-18 Z^{2}-3 Z+2=0
$$

na equação quártica desprovida do termo de segundo grau

$$
W^{4}+8 W^{3}-111 W-196=0
$$

e a mudança de variável $W=Z+1$ também transforma a equação quártica dada em uma equação quártica desprovida do termo de segundo grau

$$
W^{4}-8 W^{3}+17 W-8=0 .
$$

A escolha da mudança de variável $W=Z-1$ transforma a equação quártica dada em uma equação quártica desprovida do termo de terceiro grau

$$
W^{4}-24 W^{2}-47 W-22=0
$$


pelo dispositivo de Briot-Ruffini

$$
\begin{aligned}
& P(1)=-22, \quad P^{\prime}(1)=-47, \quad \frac{P^{\prime \prime}(1)}{2 !}=-24, \quad \frac{P^{\prime \prime \prime}(1)}{3 !}=0, \quad \frac{P^{\prime \prime \prime \prime}(1)}{4 !}=1 \\
& \hline 1 \\
& \hline 1
\end{aligned}
$$

\subsection{Algoritmo de Peletarius}

O Algoritmo de Peletarius utiliza as fórmulas derivadas para os coeficien-

\begin{tabular}{|c|c|c|c|c|c|c|}
\hline$A_{N}$ & $A_{N-1}$ & $\ldots$ & $A_{2}$ & $A_{1}$ & $A_{0}$ & \\
\hline$\underbrace{A_{N}-B_{N-1}}$ & $\frac{A_{N-1}-B_{N-2}}{Z_{0}}$ & $\cdots$ & $\frac{A_{2}-B_{1}}{Z_{0}}$ & $\frac{A_{1}-B_{0}}{Z_{0}}$ & $\frac{A_{0}-R}{Z_{0}}$ & $Z_{0}$ \\
\hline$=0$ & & & & & & \\
\hline
\end{tabular}
tes do quociente $B(Z)$ e do resto constante $R$ resultantes da divisão euclidiana de $P(Z)$ por $Z-Z_{0}$, isto é

$$
\begin{aligned}
P(Z) & =A_{N} Z^{N}+A_{N-1} Z^{N-1}+\cdots+A_{1} Z+A_{0} \\
& =\left(Z-Z_{0}\right) \cdot\left(B_{N-1} Z^{N-1}+B_{N-2} Z^{N-2}+\cdots+B_{1} Z+B_{0}\right)+R
\end{aligned}
$$

ou equivalente

$$
\begin{gathered}
A_{0}=-B_{0} Z_{0}+R \\
A_{1}=-B_{1} Z_{0}+B_{0} \\
A_{2}=-B_{2} Z_{0}+B_{1} \\
\vdots \\
A_{N-2}=-B_{N-2} Z_{0}+B_{N-3} \\
A_{N-1}=-B_{N-1} Z_{0}+B_{N-2} \\
A_{N}=B_{N-1}
\end{gathered}
$$


Impondo que $Z_{0}$ é raiz da equação polinomial

$$
P(Z)=0
$$

que é equivalente a impor que

$$
R=0
$$

$\mathrm{Ou}$

$$
A_{0}=-B_{0} Z_{0}
$$

e então

$$
\begin{gathered}
A_{1}-B_{0}=-B_{1} Z_{0} \\
A_{2}-B_{1}=-B_{2} Z_{0} \\
\vdots \\
A_{N-2}-B_{N-3}=-B_{N-2} Z_{0} \\
A_{N-1}-B_{N-2}=-B_{N-1} Z_{0}=-A_{N} Z_{0}
\end{gathered}
$$

O algoritmo de Peletarius é mais utilizado na pesquisa de soluções inteiras positivas em equações polinomiais com coeficientes inteiros: a condição $R=0$ ou $A_{0}=-B_{0} Z_{0}$ mostra que

$Z_{0}$ é um divisor inteiro de $A_{0}$

$Z_{0}$ é um divisor inteiro de $A_{1}-B_{0}$

$Z_{0}$ é um divisor inteiro de $A_{2}-B_{1}$

$Z_{0}$ é um divisor inteiro de $A_{N-2}-B_{N-3}$

$Z_{0}$ é um divisor inteiro de $A_{N-1}-B_{N-2}$

Exemplo 2.7. A equação polinomial com coeficientes inteiros

$$
Z^{4}+2 Z^{3}-19 Z^{2}-8 Z+60=0
$$

admite 2 e 3 como raízes porque pelo algoritmo de Peletarius

\begin{tabular}{lllll|l}
12 & -19 & -8 & 60 & \\
\hline 0 & $\frac{-4+2}{2}=-1$ & $\frac{11-19}{2}=-4$ & $\frac{30-8}{2}=11$ & 30 & 2
\end{tabular}




\begin{tabular}{lllll|l}
12 & -19 & -8 & 60 & \\
\hline 0 & $\frac{-5+2}{3}=1$ & $\frac{4-19}{3}=-5$ & $\frac{20-8}{3}=4$ & 20 & 3
\end{tabular}

Exemplo 2.8. A equação polinomial cúbica com coeficientes inteiros

$$
Z^{3}-29 Z^{2}+126 Z-144=0
$$

admite 2, 3 e 24 como raízes porque pelo algoritmo de Peletarius

\begin{tabular}{llll|l}
1 & -29 & 126 & -144 & \\
\hline 0 & $\frac{27-29}{2}=-1$ & $\frac{-72+126}{2}=27$ & -72 & 2 \\
1 & -29 & 126 & -144 & \\
\hline 0 & $\frac{26-29}{3}=-1$ & $\frac{-48+126}{3}=26$ & -48 & 3 \\
$1-29$ & 126 & -144 & \\
\hline 0 & $\frac{5-29}{24}=-1$ & $\frac{-6+126}{24}=5$ & -6 & 24
\end{tabular}

Exemplo 2.9. A equação polinomial cúbica com coeficientes inteiros

$$
2 Z^{3}-8 Z^{2}+15 Z-18=0
$$

não admite 3 como raízes porque pelo algoritmo de Peletarius

$$
\begin{array}{lll|l}
2-8 & 15 & -18 & \\
\hline \frac{3-8}{3}=\frac{5}{3} & \frac{-6+15}{3}=3 & -6 & 3
\end{array}
$$

obtém se $\frac{5}{3}$ o qual não é um número inteiro.

Exemplo 2.10. A equação polinomial quártica com coeficientes inteiros

$$
Z_{4}-2 Z^{3}-19 Z^{2}+8 Z+60=0
$$


não admite 3 como raízes porque pelo algoritmo de Peletarius

$$
\begin{array}{llll|l}
1-2 & -19 & 8 & 60 & \\
\hline & \frac{20+8}{3}=\frac{28}{3} & 20 & 3
\end{array}
$$

obtém se $\frac{28}{3}$ o qual não é um número inteiro.

\subsection{Fórmulas de Newton}

Lembrando as definições de

$$
\begin{gathered}
s_{0}\left(Z, Z_{1}, Z_{2}, \ldots, Z_{N}\right)=1 \\
s_{1}\left(Z, Z_{1}, Z_{2}, \ldots, Z_{N}\right)=Z_{1}+Z_{2}+. .+Z_{N} \\
=\sum_{\substack{1 \leqslant i \leqslant N-1 \\
i<j \leqslant N}} Z_{i} Z_{j} \\
=Z_{1} Z_{2}+\cdots+Z_{1} Z_{N}+Z_{2} Z_{3}+\cdots+Z_{2} Z_{N}+\cdots+Z_{N-1} Z_{N}
\end{gathered}
$$

e assim por diante

$$
s_{N}\left(Z_{1}, Z_{2}, \ldots, Z_{N}\right)=Z_{1} Z_{2} \cdots Z_{N}
$$

tem-se que $s_{0}, s_{1}, \ldots, s_{N}$, além de funções polinomiais elementares em $N$ variáveis $Z_{1}, Z_{2}, Z_{3}, \ldots, Z_{N}$, também são funções polinomiais homogêneas, isto é, para cada $k=0,1, \ldots, N s_{k}$ é homogênea de grau $k$, isto é

$$
s_{k}\left(t Z_{1}, \ldots, t Z_{n}\right)=t^{k} s_{k}\left(Z_{1}, \ldots, Z_{N}\right)
$$

Definindo

$$
\begin{aligned}
p_{1}\left(Z_{1}, \ldots, Z_{N}\right) & =s_{1}\left(Z_{1}, \ldots, Z_{N}\right)=Z_{1}+Z_{2}+\cdots+Z_{N} \\
p_{2}\left(Z_{1}, \ldots, Z_{N}\right) & =Z_{1}^{2}+Z_{2}^{2}+\cdots+Z_{N}^{2} \\
& =s_{1}\left(Z_{1}, \ldots, Z_{N}\right) p_{1}\left(Z_{1}, \ldots, Z_{N}\right)-2 s_{2}\left(Z_{1}, \ldots, Z_{N}\right) \\
& =\left[s_{1}\left(Z_{1}, \ldots, Z_{N}\right)\right]^{2}-2 s_{2}\left(Z_{1}, \ldots, Z_{N}\right) \\
p_{3}\left(Z_{1}, \ldots, Z_{N}\right) & =Z_{1}^{3}+Z_{2}^{3}+\cdots+Z_{N}^{3} \\
& =s_{1}\left(Z_{1}, \ldots, Z_{N}\right) p_{2}\left(Z_{1}, \ldots, Z_{N}\right)-s_{2}\left(Z_{1}, \ldots, Z_{N}\right)+3 s_{3}\left(Z_{1}, \ldots, Z_{N}\right)
\end{aligned}
$$




$$
\begin{aligned}
= & {\left[s_{1}\left(Z_{1}, \ldots, Z_{N}\right)\right]^{3}-s_{1}\left(Z_{1}, \ldots, Z_{N}\right) s_{2}\left(Z_{1}, \ldots, Z_{N}\right)+3 s_{3}\left(Z_{1}, \ldots, Z_{N}\right) } \\
p_{4}\left(Z_{1}, \ldots, Z_{n}\right)= & s_{1}\left(Z_{1}, \ldots, Z_{N}\right) p_{3}\left(Z_{1}, \ldots, Z_{N}\right)-s_{2}\left(Z_{1}, \ldots, Z_{N}\right) p_{2}\left(Z_{1}, \ldots, Z_{N}\right) \\
& +s_{3}\left(Z_{1}, \ldots, Z_{N}\right) p_{1}\left(Z_{1}, \ldots, Z_{N}\right)-4 s_{4}\left(Z_{1}, \ldots, Z_{N}\right) \\
= & {\left[s_{1}\left(Z_{1}, \ldots, Z_{N}\right]^{4}+4\left[s_{1}\left(Z_{1}, \ldots, Z_{N}\right)\right]^{2} s_{2}\left(Z_{1}, \ldots, Z_{N}\right)\right.} \\
& -4 s_{1}\left(Z_{1}, \ldots, Z_{N}\right) s_{3}\left(Z_{1}, \ldots, Z_{N}\right) \\
& +2\left[s_{2}\left(Z_{1}, z_{2}, \ldots, Z_{N}\right)\right]^{2}-4 s_{4}\left(Z_{1}, \ldots, Z_{N}\right)
\end{aligned}
$$

Em geral, para $1 \leqslant k \leqslant N-1$,

$p_{k}\left(Z_{1}, \ldots, Z_{N}\right)=\sum_{j=1}^{k-1}(-1)^{j+1} s_{j}\left(Z_{1}, \ldots, Z_{N}\right) p_{k-j}\left(Z_{1}, \ldots, Z_{N}\right)+(-1)^{k+1} k s_{k}\left(Z_{1}, \ldots, Z_{N}\right)$

e para $k \geqslant N$

$$
p_{k}\left(Z_{1}, \ldots, Z_{N}\right)=\sum_{j=1}^{N}(-1)^{j+1} s_{j}\left(Z_{1}, \ldots, Z_{N}\right) p_{k-j}\left(Z_{1}, \ldots, Z_{N}\right)
$$

Demonstração. O operador derivação $D$ aplicado a funções polinomiais homogêneas

$$
\begin{gathered}
D(1)=0 \\
D\left(1+Z_{j}\right)=D(1)+D\left(Z_{j}\right)=Z_{j}, j=1,2, \ldots, N \\
D\left(1+Z_{1}+Z_{3}^{2}\right)=D(1)+D\left(Z_{1}\right)+D\left(Z_{3}\right)^{2}=Z_{1}+2 Z_{3}
\end{gathered}
$$

alem de satisfazer a regra da soma e do produto satisfaz também

$$
D\left[s_{k}\left(Z_{1}, Z_{2}, \ldots, Z_{N}\right]=k s_{k}\left(Z_{1}, Z_{2}, \ldots, Z_{N}\right)\right.
$$

pelo fato de $s_{k}\left(Z_{1}, Z_{2}, \ldots, Z_{N}\right)$ ser homogênea de grau $k$.

Como

$$
\left(1+Z_{1}\right)\left(1+Z_{2}\right) \ldots\left(1+Z_{N}\right)=\sum_{k=0}^{N} s_{k}\left(Z_{1}, Z_{2}, \ldots, Z_{N}\right)
$$

$$
\sum_{1}^{N} k s_{k}\left(Z_{1}, Z_{2}, \ldots, Z_{N}\right)=D\left[\sum_{k=0}^{N} s_{k}\left(Z_{1}, Z_{2}, \ldots, Z_{N}\right)\right]
$$




$$
\begin{aligned}
= & D\left[\left(1+Z_{1}\right)\left(1+Z_{2}\right) \ldots\left(1+Z_{N}\right)\right] \\
= & \left(1+Z_{1}\right)\left(1+Z_{2}\right) \ldots\left(1+Z_{N}\right)\left(\frac{Z_{1}}{1+Z_{1}}\right)\left(\frac{Z_{2}}{1+Z_{2}}\right) \ldots\left(\frac{Z_{N}}{1+Z_{N}}\right) \\
= & \sum_{k=0}^{N} k s_{k}\left(Z_{1}, Z_{2}, \ldots, Z_{N}\right)\left[\sum_{j=0}^{\infty}(-1)^{j} Z_{1}^{j+1}\right]\left[\sum_{j=0}^{\infty}(-1)^{j} Z_{2}^{j+1}\right] \ldots\left[\sum_{j=0}^{\infty}(-1)^{j} Z_{N}^{j+1}\right] \\
= & {\left[\sum_{k=0}^{n} s_{k}\left(Z_{1}, Z_{2}, \ldots, Z_{N}\right)\right]\left[\sum_{j=1}^{\infty}(-1)^{j+1} p_{j}\left(Z_{1}, Z_{2}, \ldots, Z_{N}\right)\right] } \\
= & \sum_{k=1}^{\infty}(-1)^{k+1} p_{k}\left(Z_{1}, Z_{2}, \ldots, Z_{N}\right) \\
& \quad+\sum_{k=1}^{\infty}\left[\sum_{j=1}^{k-1}(-1)^{k-j-1} s_{j}\left(Z_{1}, Z_{2}, \ldots, Z_{N}\right) p_{k-j}\left(Z_{1}, Z_{2}, \ldots, Z_{N}\right)\right]
\end{aligned}
$$

tendo em vista que $s_{0}\left(Z_{1}, Z_{2}, \ldots, Z_{N}\right)=1$.

Pela multiplicação das componentes homogêneas de mesmo grau vem, para $k=1,2, \ldots, N-1$

$$
\begin{aligned}
& (-1)^{k+1} p_{k}\left(Z_{1}, \ldots, Z_{N}\right) \\
& +\sum_{j=1}^{k-1}(-1)^{k-1-j} s_{j}\left(Z_{1}, \ldots Z_{N}\right) p_{k-j}\left(Z_{1}, \ldots, Z_{N}\right) \\
& \quad=k s_{k}\left(Z_{1}, Z_{2} \ldots, Z_{N}\right)
\end{aligned}
$$

$\mathrm{ou}$

$$
\begin{aligned}
p_{k}\left(Z_{1}, \ldots, Z_{N}\right) & \\
=\sum_{j=1}^{k-1}(-1)^{j-1} s_{j}\left(Z_{1}, \ldots, Z_{N}\right) p_{k-j}\left(Z_{1}, \ldots, Z_{N}\right) & +(-1)^{k-1} k s_{k}\left(Z_{1}, \ldots, Z_{N}\right)
\end{aligned}
$$

tendo em vista que $(-1)^{k-1}=(-1)^{k+1}$ e para $k=N+1, N+2, \ldots$

$$
\begin{aligned}
0=(-1)^{k+1} P_{k}\left(Z_{1}, Z_{2}, \ldots, Z_{N}\right) & \\
& +\sum_{j=1}^{k-1}(-1)^{k-j-1} s_{j}\left(Z_{1}, Z_{2}, \ldots, Z_{N}\right) p_{k-j}\left(Z_{1}, Z_{2}, \ldots, Z_{N}\right)
\end{aligned}
$$


$\mathrm{ou}$

$$
p_{k}\left(Z_{1}, Z_{2}, \ldots, Z_{N}\right)=\sum_{j=1}^{k-1}(-1)^{j+1} s_{j}\left(Z_{1}, Z_{2}, \ldots, Z_{N}\right) p_{k-j}\left(Z_{1}, Z_{2}, \ldots, Z_{N}\right)
$$

Observação. Da igualdade dada pelas relações de Girard

$$
\left(Z-Z_{1}\right)\left(Z-Z_{2}\right) \ldots\left(Z-Z_{N}\right)=\sum_{k=0}^{N}(-1)^{k} s_{k}\left(Z_{1}, Z_{2}, \ldots, Z_{N}\right) Z^{N-k}
$$

colocando $Z=Z_{1}$,

$$
Z_{1}^{N}=\sum_{k=1}^{N}(-1)^{K+1} s_{K}\left(Z_{1}, Z_{2}, \ldots, Z_{N}\right) Z_{1}^{N-K}
$$

e sucessivamente $z=z_{n}$,

$$
Z_{N}^{N}=\sum_{k=1}^{N}(-1)^{k+1} s_{k}\left(Z_{1} \ldots Z_{N}\right) Z_{N}^{N-K}
$$

somando ambos os membros vem

$$
p_{N}\left(Z_{1}, Z_{2}, \ldots, Z_{N}\right)=\sum_{k=1}^{N}(-1)^{k+1} s_{k}\left(Z_{1}, Z_{2}, \ldots, Z_{N}\right) p_{N-k}\left(Z_{1}, Z_{2}, \ldots, Z_{N}\right)
$$

de

$$
Z_{1} Z_{1}^{N}=\sum_{k=1}^{N}(-1)^{k+1} s_{k}\left(Z_{1}, Z_{2}, \ldots, Z_{N}\right) Z_{N}^{N-k+1}
$$

e sucessivamente de

$$
Z_{N} Z_{N}^{N}=\sum_{k=1}^{k+1} s_{k}\left(Z_{1}, Z_{2}, \ldots, Z_{N}\right) Z_{N}^{N-k+1}
$$

e somando ambos os membros,

$p_{N+1}\left(Z_{1}, Z_{2}, \ldots, Z_{N}\right)=\sum_{k=1}^{N}(-1)^{K+1} s_{k}\left(Z_{1}, Z_{2}, \ldots, Z_{N}\right) p_{N+1-k}\left(Z_{1}, Z_{2}, \ldots, Z_{N}\right)$ 


\section{Capítulo 3}

\section{Equações polinomiais com coeficientes reais}

\subsection{Teorema da limitação das raízes reais}

As raízes reais da equação polinomial com coeficientes reais e grau $N \geqslant 1$

$$
\begin{aligned}
P(x)= & a_{N} x^{N}+a_{N-1} x^{N-1}+\cdots+a_{1} x+a_{0}=0 \\
& a_{0}, a_{1}, \ldots, a_{N-1}, a_{N} \in \mathbb{R}, a_{N} \neq 0
\end{aligned}
$$

são menores ou iguais a

- $1+\sqrt[N-R]{\max \left\{\left|\frac{a_{k}}{a_{N}}\right|\right\}}, k=0,1, \ldots, N: a_{k}<0$

em que $N-R$ é o índice do primeiro coeficiente real negativo da equação polinomial e $R>0$ é um número real arbitrário

- $2 \max \left\{\sqrt[k]{\left|\frac{a_{k}}{a_{N}}\right|}, k=0,1, \ldots, N: a_{k}<0\right\}$

em que $k$ é o índice do primeiro coeficiente negativo.

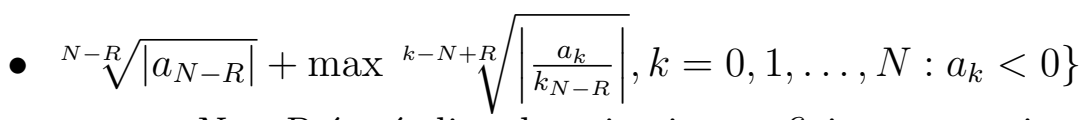
em que $N-R$ é o índice do primeiro coeficiente negativo e o índice $k$ é tomado sobre os índices dos coeficientes negativos. 


\section{Limitantes das raízes positivas}

Definição 3.1. Seja uma equação algébrica de coeficientes reais,

$$
P(x)=a_{N} x^{N}+a_{N-1} x^{N-1}+\cdots+a_{1} x+a_{0}=0
$$

com

$$
a_{0}, a_{1}, \ldots, a_{N-1}, a_{N} \in \mathbb{R}, \quad a_{N} \neq 0 .
$$

Denomina-se cota superior das raízes positivas da equação

$$
P(x)=a_{N} x^{N}+a_{N-1} x^{N-1}+\cdots+a_{1} x+a_{0}=0
$$

a um número $L$ tal que todas as raízes positivas da mesma, sejam inferiores a este número.

Denomina-se cota inferior das raízes positivas da equação

$$
P(x)=a_{N} x^{N}+a_{N-1} x^{N-1}+\cdots+a_{1} x+a_{0}=0
$$

a um número $l$, tal que todas as raízes positivas sejam superiores a este número.

Cota de Mac-Laurin $1+M$

O Método de Mac-Laurin é de grande facilidade de aplicação, conduzindo, entretanto, a uma cota, em geral, bastante elevada.

Considere a equação

$$
P(x)=a_{N} x^{N}+a_{N-1} x^{N-1}+\cdots+a_{1} x+a_{0}=0
$$

com

$$
a_{0}, a_{1}, \ldots, a_{N-1}, a_{N} \in \mathbb{R}, a_{N} \neq 0 .
$$

Chamamos $M$ o seu coeficiente negativo de maior valor absoluto concluímos que para obter uma cota superior das raízes positivas da equação $P(x)=0$, basta acrescentar uma unidade ao valor absoluto do coeficiente negativo que o tem maior. 


\section{Cota de Lagrange $1+\sqrt[n]{M}$}

Considere a equação

$$
P(x)=a_{N} x^{N}+a_{N-1} x^{N-1}+\cdots+a_{1} x+a_{0}=0
$$

e seja $a_{\alpha}$ o coeficiente negativo de maior valor absoluto e $m$ o grau do primeiro termo negativo, que supomos não ser o segundo. Obtêm-se uma cota superior das raízes positivas de uma equação somando uma unidade à raiz enésima do valor absoluto com coeficiente negativo da equação que tem maior valor absoluto $\mathrm{M}$, sendo n a diferença entre do grau da equação e do seu primeiro termo negativo.

\section{Cota de Newton}

Seja a equação $P(x)=0$, em virtude da Fórmula de Newton determinando, agora, $\mathrm{h}$ positivo de tal forma que todos os coeficientes

$$
P(h)>0, P^{\prime}(h)>0, P^{\prime \prime}(h)>0 \ldots P^{(N)}>0
$$

um valor h com essa propriedade, é uma cota superior das raízes positivas da equação considerada pois se todos os coeficientes da equação em y são positivos, ela não admite nenhuma raiz positiva.

\section{Cota de Laguerre}

O processo de Laguerre é notável pela simplicidade de aplicação e por conduzir a uma cota bastante pequena.

Seja

$$
P(x)=a_{N} x^{N}+a_{N-1} x^{N-1}+\cdots+a_{1} x+a_{0}=0
$$

dividindo o polinômio por $x-L$, sendo $L>0$.

Vem,

$$
P(x)=(x-L)\left(b_{N-1} x^{N-1}+b_{N-2} x^{N-2}+\cdots+b_{0}\right)+R
$$

onde pela regra de Ruffini,

$$
\begin{aligned}
& b_{N-1}=a_{N}>0 \\
& b_{N-2}=a_{N-1}+a_{N} L \\
& b_{N-3}=a_{N-2}+b_{N-2} L
\end{aligned}
$$




$$
R=P(L)
$$

Se o número $L$ tornar positivo todos os coeficientes $b_{N-1}, b_{N-2}, \ldots, b_{0}$ e o próprio resto $P(L)$, será uma cota superior das raízes positivas da equação dada. Será cômodo empregar o dispositivo prático para determinação do número $L$.

\section{Existência de raízes complexas}

A equação polinomial com coeficientes reais e grau $N \geqslant 2$

$$
\begin{gathered}
P(x)=a_{N} x^{N}+a_{N-1} x^{N-1}+\cdots+a_{1} x+a_{0}=0 \\
a_{0}, a_{1}, \ldots, a_{N} \in \mathbb{R}
\end{gathered}
$$

admite pelo menos um par de raízes complexas conjugadas quando

1. $a_{N-1}^{2}<2 a_{N-2}$

2. $a_{N-1}^{2}=2 a_{N-2}>0$

3. $a_{N-1}=0, a_{N-2}>0$

4. $a_{N-1}=a_{N-2}=0$

Demonstração. A prova é consequência imediata da igualdade e das relações de Girard

$$
z_{1}^{2}+z_{2}^{2}+\cdots+z_{N}^{2}=\left(z_{1}+z_{2}+\cdots+z_{N}\right)^{2}-2\left[z_{1} z_{2}+\cdots+z_{N-1} z_{N}\right.
$$

em que $z_{1}, z_{2}, \ldots, z_{N}$ são as $N$ raízes complexas da equações $P(x)=0$.

Quando $a_{N-1}^{2}>2 a_{N-2}$ não implica que a sequência polinomial admite apenas raízes reais.

Exemplo 3.2. $x^{2}+6 x+11=0$ apresenta um par de raízes complexas conjugadas e $6^{2}=36>2(11)$.

Em consequência ao fato acima, temos que

$$
P\left(\frac{1}{x}\right)=a_{N}\left(\frac{1}{x}\right)^{N}+a_{N-1}\left(\frac{1}{x}\right)^{N-1}+\cdots+a_{1}\left(\frac{1}{x}\right)+a_{0}=0
$$


com

$$
a_{0}, a_{1}, \ldots, a_{N} \in \mathbb{R}
$$

que é equivalente a escrever

$$
x^{N} P\left(\frac{1}{x}\right)=a_{0} x^{N}+a_{1} x+\cdots+a_{N-1} x^{N-1}+a_{N}=0 .
$$

De maneira análoga a equação acima admite um par de raízes complexas conjugadas quando

1. $a_{1}^{2}<2 a_{2}$

2. $a_{1}^{2}=2 a_{2}>0$

3. $a_{1}=0, a_{2}>0$

4. $a_{1}=a_{2}=0$.

O corpo $\mathbb{R}$ dos números reais não é um corpo algebricamente fechado. Uma equação polinomial de grau $N$ com coeficientes reais

$$
\begin{gathered}
P(x)=a_{N} x^{N}+a_{N-1} x^{N-1}+\cdots+a_{1} x+a_{0}=0 \\
a_{0}, a_{1}, \ldots, a_{N} \in \mathbb{R}, a_{N} \neq 0
\end{gathered}
$$

Pode admitir raízes reais e raízes complexas com parte imaginária diferente de zero. Além disso, se $a+b i \operatorname{com} b \neq 0$ é uma raiz da equação $P(x)=0$ com coeficientes reais com multiplicidade $m$ então $a-b i$, complexo conjugado de $a+b i$, também é raiz da equação $P(x)=0$ com a mesma multiplicidade $m$ e fato de

$[z-(a+b i)][z-(a-b i)]=z^{2}-[(a+b i)+(a-b i)] z+a^{2}+b^{2}=z^{2}-2 a z+a^{2}+b^{2}$

mostra que toda função polinomial de grau $N$ com coeficientes reais é fatorada como um produto de fatores lineares e de fatores quadráticos em que ocorrem coeficientes reais em todos os fatores.

$P(x)=a_{N}\left(x-x_{1}\right) \ldots\left(x-x_{s}\right)\left(x^{2}-2 a_{1} x+a_{1}^{2}+b_{1}^{2}\right) \ldots\left(x^{2}-2 a_{t} x+a_{t}^{2}+b_{t}^{2}\right)$

em que os fatores lineares $\left(x-x_{1}\right),\left(x-x_{2}\right), \ldots,\left(x-x_{s}\right)$ não são necessariamente distintos assim como os fatores quadráticos quando ocorrerem também não são necessariamente distintos. 
No caso de $x_{1}, x_{2}, \ldots, x_{s}$ serem as raízes reais distintas da equação $P(x)=$ 0 com multiplicidades $m_{1}, m_{2}, \ldots, m_{s}$ e $a_{1} \pm i b_{1}, a_{2} \pm i b_{2}, \ldots, a_{t} \pm i b_{t}$ serem as raízes complexas distintas com parte imaginária não nula com multiplicidades $n_{1}, n_{2}, \ldots, n_{t}$ então

$$
\begin{aligned}
& P(x)=a_{N}\left(x-x_{1}\right)^{m_{1}}\left(x-x_{2}\right)^{m_{2}} \ldots\left(x-x_{s}\right)^{m_{s}} \times \\
& \left(x^{2}-2 a_{1} x+a_{1}^{2}+b_{1}^{2}\right)^{n_{1}}\left(x^{2}-2 a_{2} x+a_{2}^{2}+b_{2}^{2}\right)^{n_{2}} \ldots\left(x^{2}-2 a_{t} x+a_{t}^{2}+b_{t}^{2}\right)^{n_{t}}
\end{aligned}
$$

e

$$
m_{1}+m_{2}+\cdots+m_{s}+2\left(n_{1}+n_{2}+\cdots+n_{t}\right)=N
$$

\subsection{Teorema de Bolzano-Weierstrass}

Teorema 3.3. Seja

$$
P(x)=a_{N} x^{N}+a_{N-1} x^{N-1}+\cdots+a_{1} x+a_{0}
$$

uma função polinomial de grau $N \geqslant 1$ com coeficientes reais e sejam a e $b$ números reais com $a<b$ de modo que $P(a) . P(b)<0$. Então a equação polinomial

$$
P(x)=0
$$

admite pelo menos uma raiz real c compreendida entre $a$ e $b$.

Demonstração. Sem perda de generalidade, admitindo que $P(a)<0$ e $P(b)>$ 0 (a prova para $P(a)>0$ e $P(b)<0$ é análoga), a mudança canônica de variável

$$
x=a+t(b-a)
$$

transforma a equação polinomial $P(x)=0$ dada na equação

$$
Q(t)=P[a+t(b-a)]=0
$$

em que

$$
Q(0)=P(a)<0
$$

e

$$
Q(1)=P(b)>0 .
$$


Como $Q\left(\frac{10}{10}\right)=Q(1)>0$, o conjunto $\left\{j=1,2, \ldots, 10: Q\left(\frac{j}{10}\right)>0\right\}$ de números naturais é não vazio e admite um valor mínimo: existe um valor $j_{1}$ entre 1 e 10 de modo que $Q\left(\frac{j_{1}-1}{10}\right) \leqslant 0$ e $Q\left(\frac{j_{1}}{10}\right)>0$.

$$
\begin{gathered}
\text { Definindo } c_{1}=\frac{j_{1}-1}{10} \text { e } d_{1}=c_{1}+\frac{1}{10}=\frac{j_{1}}{10} \text { vem que } d_{1}-c_{1}=\frac{1}{10} \mathrm{e} \\
Q\left(c_{1}\right) \leqslant 0 \text { e } Q\left(d_{1}\right)>0
\end{gathered}
$$

Caso $Q\left(c_{1}\right)=0$, a equação $Q(t)=0$ admite uma raiz fracionária compreendida entre 0 e 1.

Caso $Q\left(c_{1}\right)<0$ e como $Q\left(d_{1}\right)>0$ o conjunto $\left\{j=1,2, \ldots, 10: Q\left(c_{1}+\frac{j}{10^{2}}\right)>\right.$ $0\}$ é não vazio e admite um valor mínimo: existe um valor $j_{2}$ entre 1 e 10 de modo que $Q\left(c_{1}+\frac{j_{2}-1}{10^{2}}\right) \leqslant 0$ e $Q\left(c_{1}+\frac{j_{2}}{10^{2}}\right)>0$.

Definindo $c_{2}=c_{2}+\frac{j_{2}-1}{10^{2}}$ e $d_{2}=c_{1}+\frac{j_{2}}{10^{2}}$ vem que

$$
d_{2}-c_{2}=\frac{1}{10^{2}}
$$

e

$$
Q\left(c_{2}\right) \leqslant 0 \text { e } Q\left(d_{2}\right)>0
$$

Caso $Q\left(c_{2}\right)=0$, a equação polinomial $Q(t)=0$ admite uma raiz fracionária compreendida entre 0 e 1.

Caso $Q\left(c_{2}\right)<0$ e como $Q\left(d_{2}\right)>0$ o conjunto $\left\{j=1,2, \ldots, 10: Q\left(c_{2}+\frac{j}{10^{3}}\right)>\right.$ $0\}$ é não vazio e admite um valor mínimo: existe um valor $j_{3}$ entre 1 e 10 de modo que $Q\left(c_{2}+\frac{j_{3}-1}{10^{3}}\right) \leqslant 0$ e $Q\left(c_{2}+\frac{j_{3}}{10^{3}}\right)>0$.

Definindo $c_{3}=c_{2}+\frac{j_{3}-1}{10^{3}}$ e $d_{3}=c_{2}+\frac{j_{3}}{10^{3}}$ vem que

$$
d_{3}-c_{3}=\frac{1}{10^{3}}
$$

e

$$
Q\left(c_{3}\right) \leqslant 0 \text { e } Q\left(d_{3}\right)>0
$$

Caso $Q\left(c_{3}\right)=0$, a equação polinomial $Q(t)=0$ admite uma raiz fracionária compreendida entre 0 e 1. 
Caso $Q\left(c_{3}\right)<0$ e como $Q\left(d_{3}\right)>0$ o processo é repetido.

Assim por diante, ou a equação polinomial $Q(t)=0$ admite uma raiz fracionária $c_{n}$ compreendida entre 0 e 1 ou o processo é repetido indefinidamente até a obtenção de um único valor real $c$ compreendido entre as duas sequências numéricas pelo princípio dos intervalos encaixantes: Assim

$$
\begin{gathered}
c_{n}<c<d_{n} \\
d_{n}-c_{n}=\frac{1}{10^{n}} \\
Q\left(c_{n}\right)<0 Q\left(d_{n}\right)>0
\end{gathered}
$$

para $n=1,2, \ldots$

A fórmula de Taylor aplicada a função polinomial Q mostra que

$$
\begin{aligned}
& Q\left(c_{n}\right)=Q(c)+\left(c_{n}-c\right) Q^{\prime}(c)+\cdots+\left(c_{n}-c\right)^{N} \frac{Q^{(N)}(c)}{N !}<0 \\
& Q\left(d_{n}\right)=Q(c)+\left(d_{n}-c\right) Q^{\prime}(c)+\cdots+\left(c_{n}-c\right)^{N} \frac{Q^{(N)}(c)}{N !}>0
\end{aligned}
$$

ou equivalentemente

$$
\begin{gathered}
Q(c)<-\left[\left(c_{n}-c\right) Q^{\prime}(c)+\cdots+\left(c_{n}-c\right)^{N} \frac{Q^{(N)}(c)}{N !}\right] \\
Q(c)>-\left[\left(d_{n}-c\right) Q^{\prime}(c)+\cdots+\left(d_{n}-c\right)^{N} \frac{Q^{(N)}(c)}{N !}\right] .
\end{gathered}
$$

Dado um número real $\epsilon>0$ arbitrário, seja $\mathrm{M}$ o valor máximo dos módulos de $Q^{\prime}(c), \frac{Q^{\prime \prime}(c)}{2 !}, \ldots, \frac{Q^{(N)}(c)}{N !}$. Então

$$
\left|\left(c_{n}-c\right) Q^{\prime}(c)+\cdots+\left(c_{n}-c\right)^{N} \frac{Q^{(N)}(c)}{N !}\right| \leqslant M\left[\left|\left(c_{n}-c\right)\right|+\cdots+\left|\left(c_{n}-c\right)^{N}\right|\right] \leqslant \frac{M N}{10^{n}}
$$

como a mesma desigualdade é válida para $d_{n}$ no lugar de $c_{n}$, escolhendo $\mathrm{n}$ natural de modo que

como

$$
\frac{1}{10^{n}}<\frac{\epsilon}{M N}
$$

$$
0<d_{n}-c<\frac{1}{10^{n}}
$$


e

$$
0<c_{n}-c<\frac{1}{10^{n}}
$$

temos

$$
-\epsilon<Q(c)<\epsilon
$$

em que $\epsilon>0$ é um número real arbitrário.

Logo,

$$
Q(c)=0
$$

isto é, c é uma raiz irracional da equação $Q(t)=0$ compreendida entre 0 e 1.

O lema seguinte também poderia ser utilizado para a conclusão do Teorema de Bolzano-Weierstrass.

Lema 3.4. Seja $P$ a função polinomial de grau $N \geqslant 1$ com coeficientes reais dada por

$$
P(x)=a_{0}+a_{1} x+a_{2} x^{2}+\cdots+a_{N} x^{N}
$$

para cada $\epsilon>0$,

$$
-\epsilon<P(x)-P(0)<\epsilon
$$

sempre que $|x|<\frac{\epsilon}{M}$ é $|x|<1$ em que

$$
M=\max \left\{\left|a_{0}\right|,\left|a_{1}\right|, \ldots,\left|a_{N}\right|\right\}
$$

Demonstração.

$$
\begin{aligned}
|P(x)-P(0)| & =\left|a_{1} x+a_{2} x^{2}+\cdots+a_{N} x^{N}\right| \\
& \leqslant\left|a_{1}\right||x|+\left|a_{2}\right|\left|x^{2}\right|+\cdots+\left|a_{N}\right|\left|x^{N}\right| \\
& \leqslant M|x|\left[1+|x|+\cdots+|x|^{N-1}\right] \\
& \leqslant M|x| \frac{1-|x|^{N}}{1-|x|} \\
& \leqslant \frac{M|x|}{1-|x|}
\end{aligned}
$$

desde que $|x|<1$ e para cada $\epsilon>0$

$$
\frac{M|x|}{1-|x|}<\epsilon \Leftrightarrow M|x|<\epsilon(1-|x|) \Leftrightarrow|x|<\frac{\epsilon}{M+\epsilon}<\frac{\epsilon}{M} .
$$


Logo $|x|<1$ e $|x|<\frac{\epsilon}{M}$ implica que

$$
|P(x)-P(0)|<\epsilon
$$

ou equivalentemente a

$$
-\epsilon<P(x)-P(0)<\epsilon
$$

\subsubsection{Corolário do Teorema de Bolzano-Weierstrass}

Teorema 3.5. Seja $P$ uma função polinomial de grau $N \geqslant 1$ com coeficientes reais e sejam a e $b$ números reais com $a<b$.

Se $P(a) . P(b) \geqslant 0$ então a equação polinomial $P(x)=0$ admite zero ou um par de raizes reais compreendidas entre a e $b$.

Se $P(a) . P(b)<0$ então a equação polinomial $P(x)=0$ admite um número impar de raízes reais compreendidas entre a $e b$.

Demonstração. Sejam $x_{1}<x_{2}<x \cdots<x_{p}$ as raízes da equação polinomial $P(x)=0$ com multiplicidades $m_{1}, m_{2}, \ldots, m_{p}$ respectivamente, compreendidas entre $a$ e $b$.

Então

$$
P(x)=\left(x-x_{1}\right)^{m_{1}}\left(x-x_{2}\right)^{m_{2}} \ldots\left(x-x_{p}\right)^{m_{p}} S(x)
$$

em que $S(x)$ é uma função polinomial cujo sinal é constante no intervalo fechado $[a, b]$.

Logo o sinal de

$$
\frac{P(a)}{P(b)}=\left(\frac{a-x_{1}}{b-x_{1}}\right)^{m_{1}}\left(\frac{a-x_{2}}{b-x_{2}}\right)^{m_{2}} \ldots\left(\frac{a-x_{p}}{b-x_{p}}\right)^{m_{p}} \frac{S(a)}{S(b)}
$$

é o sinal de

$$
(-1)^{m_{1}+m_{2}+\cdots+m_{p}}
$$

Portanto, se $\frac{P(a)}{P(b)} \geqslant 0$ é equivalente a dizer que o número de raízes da equação $P(x)=0$ compreendidas entre a e b é um número par, possivelmente zero ou se $\frac{P(a)}{P(b)}<0$ é equivalente a dizer que o número de raízes da equação $P(x)=0$ compreendidas entre a e b é um número impar. 


\subsection{Regra de sinais de Descartes}

Teorema 3.6. O número $R$ das raízes reais positivas da equação polinomial de grau $N$ com coeficientes reais

$$
\begin{gathered}
P(x)=a_{N} x^{N}+a_{N-1} x^{N-1}+\cdots+a_{1} x+a_{0}=0, \\
a_{0}, a_{1}, \ldots, a_{N} \in \mathbb{R}
\end{gathered}
$$

(em que cada raiz real positiva é contada de acordo com a sua multiplicidade) é menor ou igual ao número $V$ de variações de sinal da sequência finita dos coeficientes da equação polinomial

$$
a_{0}, a_{1}, \ldots, a_{N-1}, a_{N}
$$

No caso do número $R$ das raízes reais positivas de $P(x)=0$ ser estritamente menor do que $V$, a diferença $V-R$ é um número natural par, ou equivalentemente, a fórmula $V=R+2 d$ para algum $d \in\{0,1,2, \ldots\}$ é válida nos dois casos a saber de igualdade entre $R$ e $V$ e da desigualdade estrita entre $R$ e $V$.

O número de raízes reais negativas de $P(x)=0$ é igual ao número de raízes reais positivas da equação $P(-x)=0$.

Prova por indução sobre o número $V$ de variações de sinal da sequência finita dos coeficientes reais da equação $P(x)=0$.

O caso $V=0$ é trivial pelo que, não havendo variação de sinal na sequência dos coeficientes, o número $R$ das raízes reais positivas é também nulo.

A hipótese de indução é que $V=R+2 d$ para algum $d \in 0,1,2, \ldots$ sempre que houver $V$ variações de sinal na sequência finita dos coeficientes da equação polinomial.

Assumindo uma equação polinomial de grau $N$ com coeficientes reais $P(x)=0$ em que ocorreu $(V+1)$ variações de sinal na sequência finita dos coeficientes da equação, ou sejam os coeficientes $a_{i}$ e $a_{j}$ com $i<j$ com sinais contrários (coeficientes entre $a_{i}$ e $a_{j}$ são nulos)

O número de variações de sinal $V+1$ é constituído por três parcelas a saber

1. o número de variações de sinal na sequência $a_{0}, a_{1}, \ldots, a_{i}$.

2. o número de variações de sinal na sequência $a_{i}, \ldots, a_{j}$ 
3. o número de variações de sinal na sequência $a_{j}, \ldots, a_{N}$.

Para cada $t \in \mathbb{R}$ compreendido entre $i$ e $j$, isto é, $i \leqslant t \leqslant j$, a equação auxiliar de GUA associada a $P(x)=0$ é

$$
P_{t}(x)=x P^{\prime}(x)-t P(x)
$$

cujos coeficientes são

$(N-t) a_{N},(N-t-1) a_{N-1}, \ldots,(j-t) a_{j}, \ldots,(j-t) a_{j}, \ldots,(i-t) a_{i}, \ldots$, e como os fatores $-t,(1-t), \ldots,(i-t)$ são todos negativos enquanto que os fatores $(j-t), \ldots,(N-t)$ são todos positivos, o número de variações de sinal da equação de GUA

$$
P_{t}(x)=0
$$

é então igual a $V$ pois na sequência

$$
(t-i) a_{i} \ldots(t-j) a_{j}
$$

não há variação de sinal em vista de $(t-i) a_{i}$ e de $(t-j) a_{j}$ terem o mesmo sinal (os termos intermediários são nulos)

Pelo teorema de GUA dado a seguir, a equação auxiliar de GUA admite pelo menos uma raiz real em cada um dos intervalos abertos

$\left(x_{1}, x_{2}\right) ;\left(x_{2}, x_{3}\right) ; \ldots ;\left(x_{p-1}, x_{p}\right)$ em que $x_{1} \leqslant x_{2} \leqslant \vdots \leqslant x_{p}$ é a sequência crescente das raízes positivas de $P(x)=0$.

Pela hipótese de indução, o número de raízes reais positivas da equação de GUA contadas as suas multiplicidades é menor ou igual a $V$.

Logo, o número de raízes reais positivas de $P(x)=0$ contadas as suas multiplicidades é no máximo uma unidade a mais que o número de raízes reais positivas da equação associada de GUA contadas as suas multiplicidades (que é menor ou igual a $V$ pela hipótese de indução) e, portanto, menor ou igual a $V+1$.

\subsection{Teorema de Gua}

Teorema 3.7. Seja $x_{1}<x_{2}<\cdots<x_{r}$ a sequência das raízes estritamente positivas e distintas da equação polinomial de grau $N$ com coeficientes reais 
$P(x)=0$ com multiplicidades $m_{1}, m_{2}, \ldots, m_{r}$ respectivamente. Então, para cada $t \in \mathbb{R}, t \neq 0$, a equação associada de Gua

$$
P_{t}(x)=x P^{\prime}(x)+t P(x)=0
$$

admite pelo menos uma raiz real em cada um dos intervalos abertos

$$
\left(x_{1}, x_{2}\right) ;\left(x_{2}, x_{3}\right) ; \ldots ;\left(x_{p-1}, x_{p}\right)
$$

Demonstração. Sejam $x_{1}<x_{2}<\cdots<x_{r}$ as $r$ raízes reais distintas e positivas da equação polinomial de grau $N$ com coeficientes reais $P(x)=0$. Se as multiplicidades de $x_{1}, x_{2}, \ldots, x_{r}$ são respectivamente $m_{1}, m_{2}, \ldots, m_{r}$, então $m_{1}+m_{2}+\cdots+m_{r}$ é o número de raízes reais positivas de $P(x)=0$.

Como demonstrado ao final do Capítulo anterior; para cada $j=1,2, \ldots, r$ $x_{j}$ é raiz real positiva da equação auxiliar de GUA

$$
P_{t}(x)=x P^{\prime}(x)+t P^{\prime}(x), \quad t \neq 0
$$

com multiplicidade $m_{j}-1$.

O número de raízes reais positivas de $P_{t}(x)=0$ é $\left(m_{1}-1\right)+\left(m_{2}-1\right)+$ $\cdots+\left(m_{r}-1\right)$ independentemente do valor não nulo de $t$.

Para cada $j=1,2, \ldots, r$,

$$
\begin{aligned}
& \lim _{x \rightarrow x_{j}-} x \frac{P^{\prime}(x)}{P(x)}=-\infty \\
& \lim _{x \rightarrow x_{j}+} x \frac{P^{\prime}(x)}{P(x)}=+\infty
\end{aligned}
$$

Isto implica que, como

$$
\begin{aligned}
& \frac{P_{t}(x)}{P(x)}=x \frac{P^{\prime}(x)}{P(x)}+t \\
& \frac{P_{t}\left(x_{j}+10^{-n}\right)}{P\left(x_{j+1}+10^{-n}\right)} \geqslant 0 \\
& \frac{P_{t}\left(x_{j}-10^{-n}\right)}{P\left(x_{j+1}-10^{-n}\right)} \leqslant 0
\end{aligned}
$$

para cada $j=1,2, \ldots,(r-1)$ e para cada $n \in \mathbb{N}$ suficientemente grande.

Logo, $P_{t}\left(x_{j}+10^{-n}\right) P_{t}\left(x_{j+1}-10^{-n}\right) \leqslant 0$ para $n \in \mathbb{N}$ suficientemente grande o que prova a existência de pelo menos uma raiz real da equação $P_{t}(x)=0$ compreendida entre $x_{j}$ e $x_{j+1}$. 
Em particular, o número de raízes reais positivas da equação auxiliar de GUA é no mínimo uma unidade a menos que o número de raízes reais positivas da equação $P(x)=0$.

Além disso, pode ser provada de uma maneira análoga que a equação auxiliar de GUA é no mínimo uma unidade a menos que o número de raízes reais negativas da equação $P(x)=0$.

Finalizando: se todas as raízes reais de $P(x)=0$ são reais então a equação associada de Gua $P_{t}=x P^{\prime}(x)+t P(x)$ com $t \in \mathbb{R}, t \neq 0$ admitirá no máximo duas raízes reais não complexas conjugadas desde que o grau da equação auxiliar de Gua $P_{t}(x)=0$ não é superior ao grau de $P(x)=0$.

Lema 3.8. Seja a uma raiz real com multiplicidade $m$ da equação polinomial de grau $N$ com coeficientes reais $P(x)=0$. Então:

a) Se $a \geqslant 0$ então

$$
\begin{aligned}
& \lim _{x \rightarrow a-} \frac{P^{\prime}(x)}{P(x)}=-\infty, \\
& \lim _{x \rightarrow a+} \frac{P^{\prime}(x)}{P(x)}=+\infty .
\end{aligned}
$$

b) Se $a<0$ então

$$
\begin{aligned}
& \lim _{x \rightarrow a-} \frac{P^{\prime}(x)}{P(x)}=+\infty, \\
& \lim _{x \rightarrow a+} \frac{P^{\prime}(x)}{P(x)}=-\infty .
\end{aligned}
$$

c) Se $a=0$, então

$$
\begin{aligned}
& \lim _{x \rightarrow 0} \frac{P^{\prime}(x)}{P(x)}=m, \\
& \lim _{x \rightarrow 0} \frac{P^{\prime}(x)}{P(x)}=m .
\end{aligned}
$$

Demonstração. Seja $a$ uma raiz real com multiplicidade $m$ da equação polinomial de grau $N$ com coeficientes reais $P(x)=0$. Por definição,

$$
P(x)=(x-a)^{m} Q(x), \quad Q(a) \neq 0
$$


$\mathrm{Ou}$

$$
\begin{aligned}
& P^{\prime}(x)=m(x-a)^{m-1}+(x-a)^{m} Q^{\prime}(x) \\
& P^{\prime}(x)=(x-a)^{m-1}\left[m+(x-a) Q^{\prime}(x)\right]
\end{aligned}
$$

Então,

$$
\begin{aligned}
& \frac{P^{\prime}\left(a+10^{-n}\right)}{P\left(a-10^{-n}\right)}=\frac{m}{-10^{-n}}+\frac{Q^{\prime}\left(a-10^{-n}\right)}{Q\left(a-10^{-n}\right)} \\
& \frac{P^{\prime}\left(a+10^{-n}\right)}{P\left(a+10^{-n}\right)}=\frac{m}{-10^{-n}}+\frac{Q^{\prime}\left(a+10^{-n}\right)}{Q\left(a+10^{-n}\right)}
\end{aligned}
$$

Para $n \in \mathbb{N}$ suficientemente grande,

$$
\frac{P^{\prime}\left(a-10^{-n}\right)}{P\left(a+10^{-n}\right)}
$$

é um número real negativo, cujo módulo é da ordem de $m 10^{n}$, enquanto que

$$
\frac{P^{\prime}\left(a+10^{-n}\right)}{P\left(a+10^{-n}\right)}
$$

é um número real positivo da ordem de $m 10^{n}$.

\subsection{Teorema de Rolle}

Teorema 3.9. Sejam $a$ e $b$, com $a<b$ duas raízes reais consecutivas $d a$ equação polinomial de grau $N$ com coeficientes reais $P(x)=0$

Então existe pelo menos uma raiz real da equação

$$
P^{\prime}(x)=0
$$

no intervalo aberto $(a, b)$.

Demonstração. Para $n \in \mathbb{N}$ suficientemente grande, os sinais de $P\left(a+10^{-n}\right)$ e de $P\left(b-10^{-n}\right)$ são iguais porque $a$ e $b$ são raízes consecutivas.

$$
\begin{aligned}
& P^{\prime}\left(a+10^{-n}\right) \geqslant 0 \\
& P\left(a+10^{-n}\right) \geqslant 0
\end{aligned}
$$




$$
\begin{aligned}
& P^{\prime}\left(b-10^{-n}\right) \leqslant 0 \\
& P\left(b-10^{-n}\right) \leqslant 0
\end{aligned}
$$

Consequentemente, $P^{\prime}\left(a+10^{-n}\right) P^{\prime}\left(b-10^{-n}\right) \leqslant 0$ para $\in \mathbb{N}$ suficientemente grande o que prova a existência de uma raiz real da equação $P^{\prime}(x)=0$ no intervalo $(a, b)$ pelo teorema de Bolzano.

O teorema de Rolle é a base de um método de separação das raízes da equação $P(x)=0$ conhecidas as raízes reais da equação derivada $P^{\prime}(x)=0$. O exemplo seguinte ilustra o método.

Exemplo 3.10. Seja

$$
P(x)=2 x^{5}-5 x^{4}+10 x^{2}-10 x+1=0
$$

Então

$$
P^{\prime}(x)=10\left(x^{4}-2 x^{3}+2 x-1\right)=10(x-1)^{3}(x+1)
$$

admite duas raízes reais distintas -1 e 1 .

temos os sinais

$$
\begin{gathered}
P(-\infty)=\lim _{x \rightarrow-\infty} P(x) \leqslant 0 \\
P(-1)=14 \geqslant 0 \\
P(1)=-2 \leqslant 0 \\
P(+\infty)=\lim _{x \rightarrow \infty} P(x) \geqslant 0
\end{gathered}
$$

exibem três variações de sinal que indicam três raízes reais simples da equação quadrática $P(x)=0$ uma em cada um dos intervalos abertos

$$
(-\infty,-1) ; \quad(-1,1) ; \quad(1,+\infty)
$$

\subsection{Corolários do Teorema de Rolle}

Corolário 3.11. Seja a equação polinomial de grau $N$ com coeficientes reais

$$
\begin{gathered}
P(x)=a_{N} x^{N}+\cdots+a_{1} x+a_{0}=0 \\
a_{0}, a_{1}, \ldots, a_{N} \neq 0
\end{gathered}
$$


Se a equação $P(x)=0$ tiver $r$ raízes reais então o número de raízes reais $d a$ equação $P^{\prime}(x)=0$ será no mínimo $r-1$ ou o número de raízes complexas com parte imaginária não nula da equação $P^{\prime}(x)=0$ é menor ou igual ao número de raízes complexas com parte imaginária não nula da equação $P(x)=0$.

Demonstração. Seja $r$ o número de raízes reais da equação $P(x)=0$ e seja $2 q\left(2 q^{\prime}\right)$ o número de raízes complexas com parte imaginária não nula de $P(x)=0\left(P^{\prime}(x)=0\right)$. Então:

$$
N=r+2 q
$$

e

$$
N-1=r^{\prime}+2 q^{\prime}
$$

Como $r^{\prime} \geqslant r-1, N-1=r^{\prime}+2 q^{\prime} \geqslant r-1+2 q^{\prime}$

$$
N=r+2 q \geqslant r+2 q^{\prime}
$$

o que implica

$$
2 q^{\prime} \leqslant 2 q
$$

Em particular, se todas as raízes de $P(x)=0$ forem reais, então todas as raízes de $P^{\prime}(x)=0$ serão reais.

Corolário 3.12. Se todas as raízes da equação polinomial de grau $N$ com coeficientes reais $P(x)=0$ forem reais então todas as raízes de $P^{\prime}(x)=0$ serão reais. Então todas as raízes de todas as derivadas $P^{(K)}(x)$ serão reais, para $K=1, \ldots, N-1$.

Se a equação $P(x)=0$ admitir pelo menos duas raízes reais distintas então a maior raiz real de $P(x)=0$ será limitante superior das raízes reais de $P^{\prime}(x)=0$, de $P^{\prime \prime}(x)=0$, de $P^{\prime \prime \prime}(x)=0$, e a menor raiz real de $P(x)=0$ será limitante inferior das raízes reais de $P^{\prime}(x)=0$, de $P^{\prime \prime}(x)=0$ de $P^{\prime \prime \prime}(x)=0$ e assim por diante.

Corolário 3.13. Sejam $x_{1} \leqslant x_{2} \leqslant \ldots \leqslant x_{n}$ as raízes reais positivas da equação polinomial de grau $N$ com coeficientes reais $P(x)=0$. Então todas as raízes da equação associada de GUA

$$
P_{t}(x)=x P^{\prime}(x)+t P(x)
$$

com $t>0$ serão reais. 
Demonstração. Para $n \in \mathbb{N}$ suficientemente grande e $t>0$

$$
\frac{P_{t}\left(10^{-n}\right)}{P\left(10^{-n}\right)}=10^{-n} \frac{P^{\prime}\left(10^{-n}\right)}{P\left(10^{-n}\right)}+t>0
$$

quando $P(0) \neq 0$ e quando $P(0)=0$

$$
\frac{P^{\prime}\left(10^{-n}\right)}{P\left(10^{-n}\right)}>0
$$

e como

$$
\begin{gathered}
\frac{P_{t}\left(x_{1}-10^{-n}\right.}{P\left(x_{1}-10^{-n}\right)}<0 \\
P\left(10^{-n}\right) P\left(x_{1}-10^{-n}\right)>0
\end{gathered}
$$

segue que $P_{t}\left(10^{-n}\right) P_{t}\left(x_{1}-10^{-n}\right)<0$, o que mostra a existência de pelo menos uma raiz real de $P_{t}(x)$ compreendida entre 0 e $x_{1}$.

Analogamente, prova-se a existência de pelo menos uma raiz real de $P_{t}(x)$ entre a menor raiz real negativa de $P(x)=0$ e zero.

Logo, $P_{t}(x)=0$ admite pelo menos $N-1$ raízes reais o que implica que todas as raízes da equação $P_{t}(x)=0$ são reais.

\subsection{Teorema de Sturm para equações polinomiais cujas raízes são simples}

Definição 3.14 (Sequência de Sturm de funções polinomiais associada a uma equação polinomial $P(x)=0$ cujas raízes são simples). Dada uma equação polinomial com coeficientes reais

$$
\begin{gathered}
P(x)=a_{N} x^{N}+\cdots+a_{1} x+a_{0}=0, \\
a_{0}, a_{1}, \ldots, a_{N} \neq 0
\end{gathered}
$$

e grau $N \geqslant 1$ cujas raízes são simples uma sequência de funções polinomiais

$$
P_{0}, P_{1}, \ldots, P_{s-1}, P_{s}
$$

é uma sequência de Sturm associada a equação $P(x)=0$ relativa ao intervalo aberto $(a, b)$ com $a<b$ quando 
1. $P_{0}(x)=P(x)$

2. se $x_{0} \in(a, b)$ e $P(x)=0$, então

(a) $P_{0}(x) P_{1}(x)<0$ quando $a<x<x_{0}<b$

(b) $P_{0}(x) P_{1}(x)>0$ quando $a<x_{0}<x<b$

3. funções polinomiais consecutivas não se anulam simultaneamente para valor algum entre $a$ e $b$

4. se para algum $j \in 1,2, \ldots, s-1$ e para algum $x \in(a, b)$

$$
P_{j}(x)=0
$$

então

$$
P_{j-1}(x) P_{j+1}(x)<0
$$

5. a função polinomial $P_{s}$ não se anula no intervalo aberto $(a, b)$ e de fato assume um sinal constante no intervalo aberto $(a, b)$.

\section{Construção de uma sequência de Sturm associada a uma equa-} ção polinomial $\boldsymbol{P}(\boldsymbol{x})=0$ relativa a $(-\infty,+\infty)$ (e consequentemente relativa a qualquer intervalo aberto $(a, b) \operatorname{com} a<b)$

- $P_{0}(x)=P(x)$

- $P_{1}(x)=P^{\prime}(x)$

$\left(P_{1}(x)\right.$ também pode ser escolhida como produto da função polinomial derivada $P^{\prime}(x)$ por uma constante real positiva)

- $-P_{2}(x)$ é o resto da divisão euclidiana de $P(x)$ por $P_{1}(x)$, isto é, $P_{0}(X)=P_{1}(x) Q_{1}(x)-P_{2}(x)$ em que $Q_{1}(x)$ é a função polinomial quociente e o grau de $P_{2}(x)$ é estritamente menor do que o grau de $P_{1}(x)$.

- $-P_{3}(x)$ é o resto da divisão euclidiana de $P_{1}(x)$ por $P_{2}(x)$ e o grau de $P_{3}(x)$ é estritamente menor do que o grau de $P_{2}(x)$ e, a menos da obtenção de uma função polinomial cujo sinal é mantido no intervalo aberto em consideração, o processo é continuado em um número finito de passos até em último caso, a obtenção de uma função polinomial constante de grau zero. 
A escolha de $P_{1}(x)=P^{\prime}(x)$ ou $P_{1}(x)=c P^{\prime}(x)$ para algum $c>0$ assegura que a propriedade 1 da definição é verificada.

Quanto à propriedade 2, suponha que

$$
P_{j-1}(x)=P_{j}(x) Q_{j}(x)-P_{j+1}(x)
$$

e que $P(\bar{x})=P_{j+1}(\bar{x})=0$ para algum $\bar{x} \in \mathbb{R}$. Logo $P_{j-1}(\bar{x})=0$ e assim retrosseguindo vem que $P_{1}(\bar{x})=P(\bar{x})=0$ o que indica que $\bar{x}$ é uma raiz múltipla de $P(x)=0$ mas, por hipótese a equação $P(x)=0$ não admite raízes múltiplas.

Quanto à propriedade 3 , de

$$
P_{j-1}(x)=P_{j}(x) Q_{j}(x)-P_{j+1}(x)
$$

suponha que $P_{j}(\bar{x})=0$ para algum $\bar{x} \in \mathbb{R}$ então

$$
P_{j-1}(\bar{x})=-P_{j+1}(\bar{x})
$$

como $P_{j-1} \neq 0$ e $P_{j+1}(\bar{x}) \neq 0$ pela propriedade 2 ,

$$
P_{j-1}(\bar{x}) P_{j+1}(\bar{x})<0 .
$$

Quanto à propriedade $4, P_{7}$ pode ser sempre escolhido como uma função polinomial não nula de grau zero: se $P_{s}(x)=0$, de

$$
P_{s-2}(x)=P_{s-1}(x) Q_{s-1}(x)-P_{s}(x)
$$

vem $P_{s-1}=\operatorname{mdc}\left(P, P_{1}\right)$ e novamente a equação $P(x)=0$ admite raízes múltiplas, o que contraria a hipótese.

Teorema 3.15. Seja a sequência de funções polinomiais de Sturm com coeficientes reais

$$
P_{0}(x)=P(x) ; P_{1}(x) ; P_{2}(x) ; \ldots ; P_{s}(x) ;
$$

associadas a equação polinomial $P(x)=0$, a qual não admite raízes múltiplas.

Se $a$ e b são números reais com $a<b$ e se $P(a) P(b) \neq 0$ então o número de raízes reais da equação $P(x)=0$ compreendidas entre a e $b$ coincide com a diferença entre o número $V(a)$ de variações de sinal da sequência de números reais

$$
P_{0}(a), \quad P_{1}(a), \quad P_{2}(a), \quad \ldots, \quad P_{s}(a)
$$

e o número $V(b)$ de variações de sinal da sequência de números reais

$$
P_{0}(b), \quad P_{1}(b), \quad P_{2}(b), \quad \ldots, \quad P_{s}(b)
$$


Demonstração. Na hipótese

$$
P(a) P_{1}(a) P_{2}(a) \ldots P_{s-1}(a) P(b) P_{1}(b) P_{2}(b) \ldots P_{s-1}(b) \neq 0 .
$$

O conjunto

$$
\begin{aligned}
\{x \in(a, b): P(x)=0\} \cup\left\{x \in(a, b): P_{1}(x)\right. & =0\} \cup \ldots \\
& \cup\left\{x \in(a, b): P_{s-1}(x)=0\right\}
\end{aligned}
$$

ou é um conjunto vazio ou é um conjunto finito cujos elementos $c_{1}, c_{2}, \ldots, c_{m}$ formam uma sequência finita estritamente crescente contida no intervalo aberto $(a, b)$

$$
a<c_{1}<c_{2}<\cdots<c_{m}<b .
$$

Escolhida uma sequência finita estritamente crescente $d_{0}=a, d_{1}, d_{2}, \ldots, d_{m-1}, d_{m}=$ $b$ de modo que

$$
d_{0}=a<c_{1}<d_{1}<c_{2}<d_{2}<c_{3}<\cdots<c_{m-1}<d_{m-1}<c_{m}<d_{m}=b
$$

$$
\begin{aligned}
V(a)-V(b) & =\left[V\left(d_{0}\right)-V\left(d_{1}\right)\right]+\left[V\left(d_{1}\right)-V\left(d_{2}\right)\right]+\cdots+\left[V\left(d_{m-1}\right)-V\left(d_{m}\right)\right] \\
& =\sum_{j=1}^{m}\left[V\left(d_{j-1}\right)-V\left(d_{j}\right)\right]
\end{aligned}
$$

\section{Cálculo de $V\left(d_{j-1}\right)-V\left(d_{j}\right)$ para o caso em que $P\left(c_{j}\right)=$ $P_{0}\left(c_{j}\right) \neq 0$}

Existe um subconjunto não vazio de índices

$$
\left\{j_{1}, j_{2}, \ldots, j_{k}\right\} \subset\{1,2, \ldots, j-1\}
$$

de modo que

$$
j_{1}<j_{2}<\cdots<j_{k} \text { e } P_{j_{1}}\left(c_{j}\right)=0, P_{j_{2}}\left(c_{j}\right)=0, \ldots, P_{j_{1}}\left(c_{j}\right)=0 ;
$$

pela propriedade 3 da sequência de Sturm

$$
j_{2}>j_{1}+1, j_{3}>j_{2}+1, \ldots, j_{k}>j_{k-1}+1
$$


Exprimindo a sequência de Sturm sob a forma

$$
\begin{aligned}
& P(x) P_{1}(x) P_{2}(x) \ldots P_{j_{1}-1}(x) P_{j_{1}}(x) P_{j_{1}+1}(x) \\
& P_{j_{1}+1}(x) P_{j_{1}+2}(x) \ldots P_{j_{2}-1}(x) P_{j_{2}}(x) P_{j_{2}+1}(x) \\
& \ldots \\
& P_{j_{k}+1}(x) P_{j_{k}+2}(x) \ldots P_{s-1}(x) P_{s}(x)
\end{aligned}
$$

o número de variações de sinal na sequência numérica

$$
P\left(d_{j-1}\right), P_{1}\left(d_{j-1}\right), \ldots, P_{j_{1}-1}\left(d_{j-1}\right)
$$

e na sequência numérica

$$
P\left(d_{j}\right), P_{1}\left(d_{j}\right), \ldots, P_{j}\left(d_{j}\right)
$$

são coincidentes tendo em vista que

$$
\begin{aligned}
& P\left(d_{j-1}\right) P\left(d_{j}\right)>0 \\
& P_{1}\left(d_{j-1}\right) P_{1}\left(d_{j}\right)>0 \\
& \ldots \ldots \ldots \ldots \ldots \ldots \ldots \\
& P_{j_{1}-1}\left(d_{j-1}\right) P_{j_{1}}\left(d_{j}\right)>0
\end{aligned}
$$

pois para cada $x$ compreendida entre $d_{j-1}$ e $d_{j}$ o produto

$$
P(x) P_{1}(x) \ldots P_{j_{1}-1}(x) \neq 0
$$

enquanto que nos termos de números abaixo

$$
\begin{gathered}
P_{j_{1}-1}\left(d_{j-1}\right), P_{j_{1}}\left(d_{j-1}\right), P_{j_{1}+1}\left(d_{j-1}\right) \\
P_{j_{1}-1}\left(d_{j}\right), P_{j_{1}}\left(d_{j}\right), P_{j_{1}+1}\left(d_{j}\right)
\end{gathered}
$$

ocorre apenas uma variação de sinal porque os sinais dos números

$$
P_{j_{1}-1}\left(d_{j-1}\right), P_{j_{1}-1}\left(c_{j}\right) \text { e } P_{j_{1}-1}\left(d_{j}\right)
$$

são iguais devido a inexistência de raízes da equação

$$
P_{j-1}(x=0)
$$

no intervalo aberto $d_{j-1}, d_{j}$ ) e os sinais dos três números

$$
P_{j_{1}+1}\left(d_{j-1}\right), P_{j_{1}+1}\left(c_{j}\right) \text { e } P_{j_{1}+1}\left(d_{j}\right)
$$


também são iguais pelo mesmo motivo, mas pela propriedade 3 da sequência de Sturm

$$
P_{j_{1}-1}\left(c_{j}\right) \cdot P_{j_{1}+1}\left(c_{j}\right)<0
$$

tendo em vista que $P_{j_{1}}\left(c_{j}\right)=0$.

Caso $P_{j_{1}}\left(c_{j}\right)<0$ então $P_{j_{1}-1}\left(d_{j-1}\right)<0$ e $P_{j_{1}-1}\left(d_{j}\right)<0$.

Caso $P_{j_{1}+1}\left(c_{j}\right)>0$ então $P_{j_{1}+1}\left(d_{j-1}\right)>0$ e $P_{j_{1}+1}\left(d_{j}\right)>0$.

Caso $P_{j_{1}-1}\left(c_{j}\right)>0$ e $P_{j_{1}+1}\left(c_{j}\right)>0$ as possibilidades para os sinais de

$$
P_{j_{1}-1}\left(d_{j-1}\right), P_{j_{1}}\left(d_{j-1}\right), P_{j_{1}+1}\left(d_{j-1}\right)
$$

são respectivamente,,++- ou,,+-- (uma única variação de sinal) e os sinais dos números

$$
P_{j_{1}-1}\left(d_{j}\right), P_{j_{1}}\left(d_{j}\right), P_{j_{1}+1}\left(d_{j}\right)
$$

são respectivamente,,++- ou,,+-- (uma única variação de sinal).

Os demais casos são semelhantes.

Um raciocínio análogo para os demais índices $j_{2}, j_{3}, \ldots, j_{s}$ correspondentes respectivamente aos demais trechos da sequência de Sturm permite a conclusão de que com a condição $P\left(c_{j}\right) \neq 0$

$$
\sum_{j=1}^{m}\left[V\left(d_{j-1}\right)-V\left(d_{j}\right)\right]=0
$$

em virtude de que para $j=1,2,3, \ldots, m$

$$
V\left(d_{j-1}\right)-V\left(d_{j}\right)=0
$$

\section{Cálculo de $\sum_{j=1}^{m}\left[V\left(d_{j-1}\right)-V\left(d_{j}\right)\right]$ para o caso em que $P\left(c_{j}\right)=$ 0 para algum $j \in\{1,2, \ldots, m\}$}

Tendo em vista que $P_{1}\left(c_{j}\right) \neq 0$ em virtude de $P\left(c_{j}\right)=0$ o caso anterior garante que o número de variações de sinal da sequência numérica

$$
P_{1}\left(d_{j-1}\right), P_{2}\left(d_{j-1}\right), \ldots P_{s-1}\left(d_{j-1}\right), P_{s}\left(d_{j-1}\right)
$$

e da sequência numérica

$$
P_{1}\left(d_{j}\right), P_{2}\left(d_{j}\right), \ldots P_{s-1}\left(d_{j}\right), P_{s}\left(d_{j}\right)
$$


são idênticos enquanto que o número de variações de sinal do par numérico

$$
P_{0}\left(d_{j-1}\right) \text { e } P_{1}\left(d_{j-1}\right)
$$

é igual a um e é igual a zero para o par numérico

$$
P_{0}\left(d_{j}\right) \text { e } P_{1}\left(d_{j}\right)
$$

isto é, o produto

$$
P_{0}\left(d_{j-1}\right) \cdot P_{1}\left(d_{j-1}\right)<0
$$

e o produto

$$
P_{0}\left(d_{j}\right) \cdot P_{1}\left(d_{j}\right)>0
$$

o que mostra que no caso de $P\left(c_{j}\right)=0$

$$
V\left(d_{j-1}\right)-V\left(d_{j}\right)=1
$$

Resumindo, a conclusão final é que

$$
V(a)-V(b)=\sum_{j=1}^{m}\left[V\left(d_{j-1}\right)-V\left(d_{j}\right)\right]
$$

consiste de tantas parcelas iguais a um quantas forem as raízes reais da equação $P(x)=0$ compreendidas entre $a$ e $b$ desde que o produto $P(a) \cdot P(b)$ é diferente de zero. 


\section{Capítulo 4}

\section{Equações Polinomiais com coeficientes inteiros}

Equações polinomiais com coeficientes racionais originam equações polinomiais com coeficientes inteiros após multiplicação da equação pelo mínimo múltiplo comum dos denominadores dos coeficientes.

\subsection{Critério de irredutibilidade de Eiseinstein para polinômios com coeficientes inteiros e grau $N$}

Teorema 4.1. (Teorema de Eiseinstein (1832-1852)) Um polinômio com coeficientes inteiros e grau $N \geqslant 1$

$$
P(x)=c_{N} x^{N}+c_{N+1}+\ldots+c_{1} x+c_{0}
$$

em que $c_{0}, c_{1}, \ldots, c_{N-1}, c_{N} \in \mathbb{Z}$ e $c_{N} \neq 0$ é um polinômio irredutível no anel $\mathbb{Z}$ dos números inteiros (isto é, não existem polinômios $Q(x)$ e $R(x)$ com coeficientes inteiros e grau maior ou igual a um de modo que $P(x)=Q(x) R(x)$ ) desde que exista um número natural primo $p \in \mathbb{N}$ com as propriedades

1. $p$ divide cada um dos coeficientes $c_{0}, c_{1}, \ldots, c_{N-1}$

2. $p$ não divide $c_{N}$

3. $p^{2}$ não divide $c_{0}$ 
Demonstração. Caso existam polinômios não constantes $Q(x)$ e $R(x)$ com coeficientes inteiros tal que

$$
Q(x)=a_{m} x^{m}+a_{m-1} x^{m-1}+\ldots+a_{1} x+a_{0}
$$

em que $a_{0}, a_{1}, \ldots, a_{m} \in \mathbb{Z}$, com $a_{m} \neq 0$ e $m \geqslant 1$

$$
R(x)=b_{n} x^{n}+b_{n-1} x^{n-1}+\ldots+b_{1} x+b_{0}
$$

em que $b_{0}, b_{1}, \ldots, b_{n} \in \mathbb{Z}$, com $b_{n} \neq 0$ e $n \geqslant 1$

de modo que $m+n=N$ e

$$
P(x)=Q(x) R(x)
$$

então

$$
c_{0}=a_{0} b_{0}
$$

e, como $p$ divide $c_{0}$ mas $p^{2}$ não divide $c_{0}$, então $p$ divide ou $a_{0}$ ou divide $b_{0}$ mas não divide ambos; sem perda de generalidade, admitindo que $p$ divide $a_{0}$ e $p$ não divide $b_{0}$ e por outro lado, como $p$ não divide

$$
c_{N}=a_{m} b_{n}
$$

$p$ não divide nem $a_{m}$ nem $b_{n}$ logo existe um menor índice natural $j \mathrm{com}$ $1 \leqslant m<N$ com a propriedade de que

$$
\begin{gathered}
p \text { não divide } a_{j} \\
p \text { divide } a_{0}, a_{1}, \ldots, a_{j-1}
\end{gathered}
$$

da hipótese de que

$$
p \text { divide } c_{j}=a_{0} b_{j}+a_{1} b_{j-1}+\ldots+a_{j-1} b_{1}+a_{j} b_{0}
$$

(na fórmula de $c_{j}, b_{k}=0$ quando $k>n$ ) vem que

$$
p \text { divide o produto } a_{j} b_{0}
$$

sem que $p$ divida $b_{0}$ nem $a_{j}$, o que é uma contradição ao fato de $\mathrm{p}$ ser um número natural primo.

A contradição nasceu da hipótese de que

$$
P(x)=Q(x) R(x)
$$

em que $Q(x)$ e $R(x)$ são polinômios não constantes com coeficientes inteiros. 
A conclusão final é que a existência de um número natural primo $p$ com as propriedades acima enumeradas implica na irredutibilidade do polinômio $P(x)$ em relação ao anel $\mathbb{Z}$ dos números inteiros.

Polinômios não constantes e irredutíveis no anel $\mathbb{Z}$ dos números inteiros não admitem raízes inteiras.

Os polinômios ciclotômicos, em que $p$ é um número natural primo, definidos por

$$
\Phi_{p}(x)=x^{p-1}+x^{p-2}+\ldots+x+1
$$

são polinômios irredutíveis em $\mathbb{Z}$ porque

$$
\begin{aligned}
\Phi_{p}(x+1)=\frac{(x+1)^{p}-1}{x+1-1} & \\
= & \left(\begin{array}{l}
p \\
1
\end{array}\right)+\left(\begin{array}{l}
p \\
2
\end{array}\right) x+\ldots+\left(\begin{array}{c}
p \\
p-1
\end{array}\right) x^{p-2}+x^{p-1}
\end{aligned}
$$

é um polinômio irredutível em $\mathbb{Z}$ com coeficientes inteiros devido ao critério de irredutibilidade de Eiseinstein e portanto a equação

$$
x^{p-1}+x^{p-2}+\ldots+x+1=0
$$

em que $p$ é um número natural primo, não admite raízes inteiras: as raízes dessa equação ou são irracionais ou são complexas duas a duas conjugadas.

O critério de irredutibilidade para polinômios não constantes com coeficientes racionais no corpo $\mathbb{Q}$ dos números racionais é reduzido ao critério de irredutibilidade para polinômios não constantes com coeficientes inteiros em $\mathbb{Z}$ devido ao seguinte resultado:

Teorema 4.2. Uma condição necessária e suficiente para que um polinômio não constante com coeficientes inteiros e de grau $N \geqslant 1$

$$
P(x)=c_{N} x^{N}+c_{N-1} x^{N-1}+\ldots+c_{1} x_{0}+c_{0} \operatorname{com}\left(c_{N} \neq 0\right)
$$

ser irredutivel como polinômio de coeficientes inteiros é que

1. $P(x)$ é um polinômio irredutível no corpo $\mathbb{Q}$ dos números racionais.

2. $P(x)$ é um polinômio primitivo, isto é, os coeficientes $c_{0}, c_{1}, \ldots, c_{N-1}, c_{N}$ são relativamente primos. 
Teorema 4.3 (Teorema de existência de raízes racionais para polinômios com coeficientes inteiros). Seja $\frac{a}{b}$ com $a \in \mathbb{Z}=\{\ldots,-2,-1,0,1,2, \ldots\} e$ $b \in \mathbb{N}=1,2,3, \ldots$ uma fração irredutivel (isto é, a e $b$ não apresentam fatores primos em comum) que é raiz da equação polinomial com coeficientes inteiros e grau $N \geqslant 1$.

$$
\begin{gathered}
P(x)=c_{N} x^{N}+c_{N-1} x^{N-1}+\ldots+c_{1} x+c_{0}, \text { em que } \\
c_{0}, c_{1}, \ldots, c_{N-1}, \quad c_{N} \in \mathbb{Z}, \quad c_{N} \neq 0 .
\end{gathered}
$$

Então a é um divisor inteiro de $c_{0}$ e b é um divisor inteiro de $c_{N}$.

Demonstração. A prova será feita para uma equação polinomial com coeficientes inteiros e grau três

$$
P(x)=c_{3} x^{3}+c_{2} x^{2}+c_{1} x+c_{0}
$$

Se

$$
P\left(\frac{a}{b}\right)=c_{3}\left(\frac{a}{b}\right)^{3}+c_{2}\left(\frac{a}{b}\right)^{2}+c_{1}\left(\frac{a}{b}\right)+c_{0}=0
$$

então

$$
a\left(c_{3} a^{2}+c_{2} a b+c_{1} b^{2}\right)=-c_{0} b^{3}
$$

e, como $a$ e $b$ são primos entre si, a é um divisor de $c_{0}$; de

$$
-c_{3} a^{3}=b\left(c_{2} a^{2}+c_{1} a b+c_{0} b^{2}\right)
$$

vem pela mesma razão que b é um divisor de $c_{3}$.

\subsection{Regras de exclusão de Newton}

Um método direto para o cálculo das raízes racionais de uma equação polinomial com coeficientes inteiros e grau $N \geqslant 1$ é que se

$$
P\left(\frac{a}{b}\right)=0, \text { com } a \neq 0
$$

o resto da divisão euclidiana de $P(x)$ por $b x-a$ é nulo, isto é,

$$
P(x)=(b x-a) Q(x)
$$

e, consequentemente, de

$$
P(1)=(b-a) Q(1)
$$


e de

$$
P(-1)=-(b+a) Q(-1)
$$

vem que $b-a$ é um divisor inteiro de $P(1)$ e que $b+a$ é um divisor inteiro de $P(-1)$.

Um método direto para o cálculo das raízes inteiras de uma equação polinomial com coeficientes inteiros e grau $N \geqslant 1$ é que se

$$
P(m)=0
$$

em que $\mathrm{m}$ é um número inteiro não nulo então o resto da divisão euclidiana de $P(x)$ por $x-m$ é nulo, isto é,

$$
P(x)=(x-m) Q(x)=-(m-x) Q(x)
$$

e, em consequência, de

$$
P(1)=-(m-1) Q(1)
$$

vem que $m-1$ é um divisor inteiro de $P(1)$ e de

$$
P(-1)=-(m+1) Q(-1)
$$

vem que $m+1$ é um divisor inteiro de $P(-1)$.

Estas condições necessárias para a existência de raízes inteiras para equações polinomiais com coeficientes inteiros são conhecidas como regras de exclusão de Newton:

Toda raiz inteira $m$ não nula de uma equação polinomial com coeficientes inteiros

$$
P(x)=0
$$

deve ser tal que

$$
\begin{gathered}
m-1 \text { é um divisor inteiro de } P(1) \\
m+1 \text { é um divisor inteiro de } P(-1)
\end{gathered}
$$

\subsection{Critérios de Gauss para a existência de raízes inteiras}

O critério de Gauss para a existência de raízes inteiras não nulas em equações polinomiais com coeficientes inteiros

$$
P(x)=0
$$


decorre do fato de que se m é um número inteiro não nulo e se

$$
P(x)=-(m-x) Q(x)
$$

então de

$$
\begin{gathered}
P(1)=-(m-1) Q(1) \\
P(0)=-m Q(0) \\
P(-1)=-(m+1) Q(-1)
\end{gathered}
$$

vem que o produto dos números inteiros

$$
P(1) P(0) P(-1)
$$

é um múltiplo inteiro de três.

O critério de Gauss é mais utilizado como uma regra para não existência de raízes inteiras em equações polinomiais com coeficientes inteiros

$$
P(x)=0 .
$$

Se o produto

$$
P(-1) P(0) P(1)
$$

não for um múltiplo inteiro de três então a equação polinomial $P(x)=0$ não admitirá raízes inteiras.

Mudanças de variável da forma $y=k x$ transformam a pesquisa de raízes racionais em equações polinomiais com coeficientes na pesquisa de raízes inteiras em equações polinomiais com coeficientes inteiros.

Exemplo 4.4. A mudança de variável $y=k x$, em que $k$ é um número natural, transforma a equação quártica com coeficientes inteiros

$$
3 x^{4}-4 x^{3}-19 x^{2}+8 x+12=0
$$

na equação

$$
\frac{3}{k^{4}}\left[y^{4}-\frac{k}{3} y^{3}-\frac{19}{3} k^{2} y^{2}+\frac{8}{3} k^{3} y+\frac{12}{3} k^{4}\right]=0
$$

e a escolha de $k=3$ acarreta na equação

$$
y^{4}-4 y^{3}-57 y^{2}+72 y+324=0
$$


cujas possíveis raízes inteiras são os divisores inteiros de 324 e cujas possíveis raízes naturais são

$$
1,2,3,4,6,9,12,18,27,36,54,81,108,162,324
$$

mas como a cota superior das raízes positivas desta equação calculada pelo método de Laguerre é 10, portanto as possíveis raízes naturais são apenas

$$
1,2,3,4,6 e 9
$$

e tendo em vista as regras de exclusão de Newton,

$$
P(1)=336 \text { e } P(-1)=200
$$

reduzem as candidatas das raízes nos números

$$
3,4 e 9
$$

Por inspeção, conclui-se que 3 e 9 são raízes da equação

$$
y^{4}-4 y^{3}-57 y^{2}+72 y+324=0
$$

o que é equivalente a dizer que 1 e 3 são raízes da equação

$$
3 x^{4}-4 x^{3}-19 x^{2}+8 x+12=0 .
$$

Como

$$
\begin{aligned}
y^{4}-4 y^{3}-57 y^{2}+72 y+324 & =(y-3)(y-9)\left(y^{2}+8 y+12\right) \\
& =(y-3)(y-9)(y+2)(y+6)
\end{aligned}
$$

e

$$
\begin{aligned}
3 x^{4}-4 x^{3}-19 x^{2}+8 x+12 & =3(x-1)(x-3)(x+2)\left(x+\frac{2}{3}\right) \\
& =(x-1)(x-3)(x+2)(3 x+2)
\end{aligned}
$$

o que mostra que $P(x)$ é um polinômio redutível no anel $\mathbb{Z}$ dos números inteiros e no corpo $\mathbb{Q}$ dos números racionais.

O exemplo seguinte não utiliza a mudança de variável e aplica o método direto para a pesquisa das raízes racionais em equações polinomiais com coeficientes inteiros. 
Exemplo 4.5. As possíveis raízes racionais da equação cúbica com coeficientes inteiros

$$
P(x)=6 x^{3}-31 x^{2}+30 x-8=0
$$

são da forma $\frac{a}{b}$ em que $a$ é um divisor inteiro de 8 e $b$ é um divisor inteiro de 6 , isto é, as possíveis raízes fracionárias positivas da equação dada são

$$
\frac{1}{1}, \frac{1}{2}, \frac{1}{3}, \frac{1}{6}, \frac{2}{1}, \frac{2}{2}, \frac{2}{3}, \frac{2}{6}, \frac{4}{1}, \frac{4}{2}, \frac{4}{3}, \frac{4}{6}, \frac{8}{1}, \frac{8}{2}, \frac{8}{3}, \frac{8}{6} .
$$

Como a cota superior de Newton para as raízes positivas da equação é igual a 5, as regras de exclusão de Newton serão aplicadas as frações

$$
\frac{1}{1}, \frac{2}{1}, \frac{2}{2}, \frac{2}{6}, \frac{4}{1}, \frac{4}{2}, \frac{4}{6}, \frac{8}{1}, \frac{8}{2}, \frac{8}{6} \text {. }
$$

De $P(1)=-3$ e $P(-1)=-75$ vem que se $P\left(\frac{a}{b}\right)=0$

$$
\begin{gathered}
a-b \text { é divisor inteiro de }-3 \\
a+b \text { é divisor inteiro de }-75
\end{gathered}
$$

o que reduz as candidatas a raiz às frações

$$
\frac{1}{2} \mathrm{e} \frac{2}{3}
$$

Por inspeção, $\frac{1}{2}$ e $\frac{2}{3}$ são raízes de $P(x)=0$ e

$$
\begin{aligned}
P(x)=6 x^{3}-31 x^{2}+30 x-8 & =\left(x-\frac{1}{2}\right)\left(x-\frac{2}{3}\right)(6 x-24) \\
& =(2 x-1)(3 x-2)(x-4)
\end{aligned}
$$

o que mostra que $P(x)$ é um polinômio redutível no corpo dos números racionais e que $P(x)$ é um polinômio redutível no anel $\mathbb{Z}$ dos números inteiros assim como no corpo $\mathbb{R}$ dos números reais.

Para comparar os métodos apresentados, o exemplo seguinte utiliza mudança de variável para a pesquisa de raízes racionais em equações polinomiais com coeficientes inteiros. 
Exemplo 4.6. A mudança e variável y=6x transforma a equação cúbica com coeficientes inteiros

$$
P(x)=6 x^{3}-29 x^{2}+21 x-4=0
$$

na equação cúbica

$$
y^{3}-29 y^{2}+126 y-144=0
$$

cujas possíveis raízes naturais positivas são os 15 divisores de 144, que são eles:

$$
1,2,3,4,6,8,9,12,16,18,24,36,48,72 \text { e } 144
$$

mas a cota superior das raízes positivas calculadas pelo método de Laguerre é 29 o que reduz as candidatas a raiz aos números

$$
1,2,3,4,6,8,9,12,16,18 \text { e } 24
$$

e as regras de exclusão de Newton, observando que $P(-1)=-300$ e $P(1)=$ -46 , mostram que as possíveis raízes naturais são

$$
2,3 \text { e } 24 \text {. }
$$

Por inspeção, 2, 3 e 24 são as raízes de

$$
y^{3}-29 y^{2}+126 y-144=(y-2)(y-3)(y-24)=0
$$

e que $\frac{2}{6}, \frac{3}{6}$ e $\frac{24}{6}$ são as raízes da equação cúbica dada

$$
\begin{aligned}
P(x)=6 x^{3}-29 x^{2}+21 x-4 & =6\left(x-\frac{1}{3}\right)\left(x-\frac{1}{2}\right)(x-4) \\
& =(3 x-1)(2 x-1)(x-4)
\end{aligned}
$$

Portanto $P(x)$ é um polinômio redutível no anel $\mathbb{Z}$ dos números inteiros e no corpo $\mathbb{Q}$ dos números racionais assim como no corpo $\mathbb{R}$ dos números reais. 


\section{Referências Bibliográficas}

[1] USPENSKY, J. V., Theory of Equations, Mc Grawhull Book Company Ine, 1948.

[2] TIGNOL, J-P, Galois' Theory of Algebraic Equations, Longmen Scientific and Technical, 1987.

[3] SERrÃo, A. N., Exercícios e Problemas de Álgebra, Ao Livro Técnico Rio de Janeiro, 1986.

[4] FADEEV, D. K. e SOMINSKII, I. S., Problems in Higher Algebra, W.H. Freeman and Company, San Francisco London, 1965.

[5] HARTSHORNE R., Geometry: Euclid And Beyond, Springer, Undergraduate texts in mathematics, 2000.

[6] MUtAFian C., Équations Algébriques et Théorie de Galois, Thèmes Vuibert Université Mathématiques, 1980. 